\title{
Rate-Distortion and Outage Probability Analyses for Single Helper Assisted Lossy Communications
}

\author{
Wensheng Lin, Student Member, IEEE, Qiang Xue, Member, IEEE, Jiguang He, Student Member, IEEE, \\ Markku Juntti, Senior Member, IEEE, and Tad Matsumoto, Fellow, IEEE
}

\begin{abstract}
The primary objective of this paper is to investigate the performance improvement provided by a helper, with the aim of applications to a lossy communication system in wireless sensor networks (WSNs) and/or Internet of Things (IoT). Initially, we formulate the system model as a problem of multiterminal source coding with a helper, and determine an inner bound on the achievable rate-distortion region for binary sources. Based on Shannon's lossy source-channel separation theorem, we further derive the outage probability of the system over block Rayleigh fading channels. The numerical results indicate that a helper can obviously expand the achievable rate-distortion region and provide diversity gains which in turn decrease the outage probability. Finally, we evaluate the practical performance of outage probability through simulations with exclusive-or (XOR) as an example of the helper structure. Both the simulation and theoretical results show second order diversity by introducing a helper, and lower outage probability by increasing the acceptable distortions.
\end{abstract}

Index Terms-Wireless sensor network, Internet of Things, rate-distortion, outage probability, Rayleigh fading.

\section{INTRODUCTION}

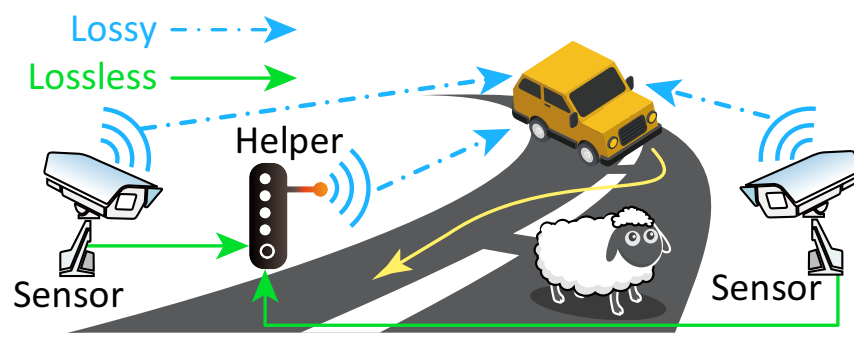

Fig. 1. A scenario of lossy communications with a helper.

In smart society, sensors are widely deployed to detect and monitor the objects, the information of which is exchanged

Copyright (c) 2015 IEEE. Personal use of this material is permitted. However, permission to use this material for any other purposes must be obtained from the IEEE by sending a request to pubs-permissions@ieee.org.

Corresponding author: Wensheng Lin.

This work is funded in part by China Scholarship Council (CSC) and in part by JAIST Core-to-Core Program. This work has been also performed in part under JSPS Kakenhi (B)15H04007, and in part by Academy of Finland 6Genesis Flagship (grant 318927).

W. Lin is with the School of Information Science, Japan Advanced Institute of Science and Technology, Ishikawa 923-1292, Japan (email: linwest@jaist.ac.jp).

Q. Xue, J. He and M. Juntti are with the Centre for Wireless Communications, University of Oulu, Oulu FI-90014, Finland (email: qiang.xue@oulu.fi; jiguang.he@oulu.fi; markku.juntti@oulu.fi)

T. Matsumoto is with the School of Information Science, Japan Advanced Institute of Science and Technology, Ishikawa 923-1292, Japan, and also with the Centre for Wireless Communications, University of Oulu, Oulu FI-90014, Finland (email: matumoto@jaist.ac.jp) via Internet of Things (IoT) [1]. For instance, as illustrated in Fig. 1, there are some sensors detecting objects on the road to provide support information for driving control of vehicles. Due to the diverse positions and detecting angles of sensors, their observations contain correlated information which can be utilized in joint decoding. In order to further improve the system performance, the concept of helper has been introduced into diverse communications systems to provide side information [2]-[5]. Besides, the relay in practical systems could also be regarded as a helper, which knows the source information (either fully or partially) and generates its own information to assist transmissions. For instance, Joda and Lahouti [6] apply a broadcasting relay at orthogonal two-hop network and design the network code at the relay; He et al. [7] adopt an exclusive-OR (XOR) operation at the relay in non-orthogonal multiple access relay channel (MARC). Likewise, a helper is located in the wireless sensor network (WSN) as shown in Fig. 1, to assist transmissions and make communications more reliable ${ }^{1}$. It should be noted here that, in this paper, the concept of "helper" represents a general device which collects information only from the sensors, while the sensors do not communicate with each other. For simplicity, we assume that the helper can access the sensor observations through errorfree channels, while the transmissions to the vehicle suffer from block Rayleigh fadings. The case where the helper can only know the lossy version of the sensor observations is left as the future work.

Due to the time varying nature of channel conditions in vehicular networks resulted from fading, the final objective of this paper is to derive the outage probability analytically. In IoT, since the sensor observations are exploited to make a final judgement other than achieve lossless reconstruction, the received information sequences are still valuable if the distortions are within a certain set of acceptable degrees. Therefore, in this paper, the outage event is defined as that the recovered observations cannot satisfy the specified distortion requirements of both the sensor observations. Regarding the calculation of theoretical outage probability, we can refer to previous works presented in [8], [9], where the outage probability is investigated for lossless communications with a lossy-forwarding (LF) relay. The calculation of the theoretical outage probability based on the achievable rate region and Shannon's source-channel separation theorem [10], [11]. First,

\footnotetext{
${ }^{1}$ Introducing a helper may make the joint decoder more complicated and consume more power; however, this paper only focuses on the performance improvement provided by a helper, while the trade-off between power consumption and performance enhancement is out of the scope.
} 
given the channel capacity and the channel coding rate, the source coding rate is determined. Then, the outage probability is the integral with respect to the multiple links' instantaneous signal-to-noise ratios (SNRs) that cannot support the source coding rates all falling inside the achievable rate region. Following the same logic, for the theoretical performance analysis of the system depicted in Fig. 1, we can start from the ratedistortion analysis of a multiterminal source coding problem. Then, based on Shannon's lossy source-channel separation theorem for multiterminal communications [12], the results of rate-distortion analysis can be further utilized in the derivation of outage probability in block fading channels.

It should be noticed that, Shannon's lossy source-channel separation theorem states that the source sequence can be compressed until a certain rate by lossy source coding, such that the lossy-encoded codeword can be losslessly transmitted through the channel, given an instantaneous SNR. Here, channel coding is regarded as a method for supporting reliable transmission of the lossy-encoded codeword generated by source coding, while the distortions occurring at the decoder output is due to the use of lossy source coding. We can then easily derive the mathematical relationship between the channel capacity and the distortions. Actually, in a practical system, the distortions is due to the use of lossy source coding and/or the transmission through channel.

If sensors do not perform lossy source coding, distortions can only be caused in the transmission through the channels. Since the instantaneous channel capacity itself is random variable due to fading, sensors do not know the instantaneous channel capacity. Hence, they are not able to know whether the lossy-encoded codeword can be transmitted losslessly over the channel. This indicates that even though the error occurrence process in the practical systems may be different from what Shannon's lossy source-channel separation theorem states, the outage calculation described above can still be used, and the analytically derived results can be used as reference.

Regarding the rate-distortion analysis, we are inspired by previous researches belonging to the category of multiterminal source coding. Slepian and Wolf first established the theoretical basis of distributed source coding in [13], where the admissible rate region is determined for losslessly recovering two discrete memoryless sources. Surprisingly, even though the encoders with multiple sources do not communicate with each other, the admissible rate region is still the same as in the case where the encoders communicate with each other. For the lossy case, Berger [14] and Tung [15] derived the outer and inner bounds on the rate-distortion region for distributed lossy compression systems, where the recovered data sequence deviates from the original sequence at a distortion level specified by the rate-distortion region. When solving the binary chief executive officer (CEO) problem, He et al. [16] derived an outer bound of the rate-distortion region for binary multiterminal source coding. Then, the outer bound was extended to the case with arbitrary number of binary sources in [17].

Moreover, the system investigated in this paper contains a helper, providing compressed side information. Therefore, we can also obtain hints from the research achievements for efficient exploitation of side information or a helper. In [18], Ahlswede and Korner determined the rate region of the lossless source coding problem with a helper. Wyner and Ziv [19] presented the result of their pioneering work on lossy compression with side information. The Wyner-Ziv theorem implies that the system with noncausal side information only available at the decoder requires a lower transmission rate than the system without side information, despite encoding without noncausal side information. Sechelea et al. in [20] analyzed the problem of lossy compression with binary sources and correlated side information in depth. For the case with more than one source, Wagner and Anantharam [21] studied a multiterminal source coding problem with one link of uncompressed side information available. In [22], Jana and Blahut derived the bonds for lossless and lossy multiterminal source coding systems where lossless and lossy links are mixed. Timo et al. [23] derived an upper bound on the rate-distortion function for lossy source coding with various side information utilized in many decoders.

So far, there are also a number of research works related to outage probability analysis for communications with correlated information. Laneman et al. characterized the outage probabilities of amplify-and-forward (AF) and decode-andforward (DF) relaying strategies for Rayleigh fading channels in [24], where the relay and source messages can be regarded as correlated information. Zhou et al. [25] derived the outage probability for the system with two correlated binary sources communicating through orthogonal MARC over block Rayleigh fadings. In [26], Lu et al. analyzed the outage probability of the MARC system where two correlated sources suffer from block Rayleigh fadings, and the estimate of source in the relay may contain errors.

Nevertheless, the outage probability is still unknown for lossy end-to-end multiterminal communications with a helper. In order to conduct the outage probability analysis, it is necessary to determine the achievable rate-distortion region for multiterminal source coding with a helper. Notice that the exact achievable rate-distortion region for lossy multiterminal source coding is still an open problem. Thus, for the achievable rate-distortion region used in the derivation of outage probability, we only consider the inner bound, i.e., the lossy recoveries must satisfy the distortion requirements if the link rates are larger than the inner bound.

The contributions of this paper are summarized as follows:

- We derive an inner bound on the achievable ratedistortion region of lossy multiterminal source coding problem with two binary sources and a helper.

- Then, we calculate the outage probabilities base on the derived inner bound and Shannon's lossy source-channel separation theorem.

- By utilizing the derived mathematical results, we conduct an in-depth investigation of performance improvement by introducing a helper. It is remarkable that a helper can not only enlarge the achievable rate-distortion region, but also provide diversity gains and reduce the outage probability.

- In addition, we verify the tendency of theoretical results through simulations, in which a joint decoding algorithm is designed for the helper sequence generated by XOR 
operation. The system can achieve second order diversity by introducing a helper, and have lower outage probability for less strict distortion requirements, both in the simulations and the theoretical analysis.

The remainder of this paper is organized as follows. Section II states the mathematical details of multiterminal source coding with a helper and the channel model. In Section III, we analyze the trade-off between link rates and the distortions remaining in the recoveries. Subsequently, Section IV derives the outage probability by utilizing the inner bound on the achievable rate-distortion region. We then conduct a series of simulations to evaluate the practical system performance for comparison with theoretical results in Section V. Finally, we conclude this work in Section VI.

\section{SySTEM MOdEL}

Notations: The random variables and their realizations are denoted by uppercase and lowercase letters, respectively. Calligraphic letters $\mathcal{X}, \mathcal{Y}, \mathcal{M}$ and $\mathcal{Q}$ denote the finite alphabets of a variable. For a random variable and its realization, we use $i$ to denote the time index, and the superscript to denote the length of the vector. In particular, we use $j \in\{1,2\}$ and " $\mathrm{H}$ " at the subscript to represent the link index for two sources and the helper, respectively.

As stated above, the performance analysis of lossy communications with a helper follows two steps, i.e., the derivation of the achievable rate-distortion region, and the calculation of outage probability. In this section, we formulate the mathematical framework of multiterminal source coding with a helper, and introduce the channel model to be used in the derivation of outage probability.

\section{A. Multiterminal Source Coding with a Helper}

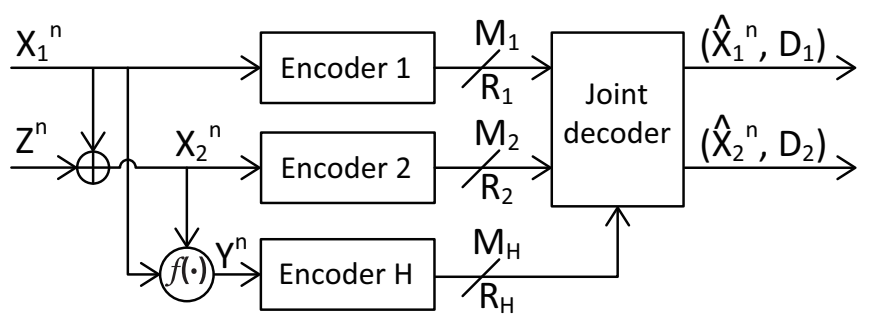

Fig. 2. The theoretical model of multiterminal source coding problem with two sources and one helper providing compressed side information.

Since the sensor observations and the helper information are transmitted in the form of binary packets, they can be regarded as correlated binary sources. We consider the simplest case that there are only two sensors in the system as illustrated in Fig. 2. The sensor observations are assumed to be two independent and identically distributed (i.i.d.) sequences $x_{1}^{n}=\left\{x_{1}(i)\right\}_{i=1}^{n}$ and $x_{2}^{n}=\left\{x_{2}(i)\right\}_{i=1}^{n}$, generated by two correlated discrete memoryless sources $X_{1}$ and $X_{2}$, respectively. At $i$-th time slot, $x_{j}(i)$ takes values from the binary alphabet $\mathcal{X}_{j}=\{0,1\}$ for $j=1,2$. Thus, $X_{2}$ is equivalent to the output of a binary symmetric channel (BSC) with input $X_{1}$ and crossover probability $\rho$ and vice versa, i.e., $X_{2}=X_{1} \oplus Z$ with $Z \sim \operatorname{Bern}(\rho)$.
In this paper, we consider the sources $X_{j} \sim \operatorname{Bern}(0.5)$ for $j=1,2$, with the joint probability mass function (PMF) $p_{X_{1}, X_{2}}\left(x_{1}, x_{2}\right)=\operatorname{Pr}\left\{X_{1}=x_{1}, X_{2}=x_{2}\right\}$ given by

$$
p_{X_{1}, X_{2}}\left(x_{1}, x_{2}\right)= \begin{cases}\frac{1}{2} \rho, & \text { if } x_{1} \neq x_{2}, \\ \frac{1}{2}(1-\rho), & \text { otherwise. }\end{cases}
$$

Notice that $X_{1}^{n}$ and $X_{2}^{n}$ are correlated sequences, and hence the helper should accumulate the information to avoid too much redundancy ${ }^{2}$. Since the helper sequence $y^{n}=\{y(i)\}_{i=1}^{n}$ highly depends on the helper structure, we assume without loss of generality that $Y$ is a function $f(\cdot)$ of $X_{1}$ and $X_{2}$.

To begin with, three sequences $x_{1}^{n}, x_{2}^{n}$ and $y^{n}$ are independently encoded by encoder 1 , encoder 2 and encoder $\mathrm{H}$ at coding rates $R_{1}, R_{2}$ and $R_{\mathrm{H}}$, respectively. The encoding process can be performed by assigning an index $M$ to each sequence according to the following mapping rules:

$$
\begin{aligned}
\varphi_{j}: \mathcal{X}_{j}^{n} \mapsto \mathcal{M}_{j} & =\left\{1,2, \cdots, 2^{n R_{j}}\right\} \text { for } j=1,2, \\
\varphi_{\mathrm{H}}: \mathcal{Y}^{n} \mapsto \mathcal{M}_{\mathrm{H}} & =\left\{1,2, \cdots, 2^{n R_{\mathrm{H}}}\right\},
\end{aligned}
$$

where the numbers of codewords are $2^{n R_{j}}$ for $X_{j}$ and $2^{n R_{\mathrm{H}}}$ for $Y$. If we separately focus on only one link, $R_{j}<H\left(X_{j}\right)$ and $R_{\mathrm{H}}<H(Y)$ are lossy compression, while $R_{j} \geq H\left(X_{j}\right)$ and $R_{\mathrm{H}} \geq H(Y)$ are lossless compression. Generally, the source coding rate is assumed to be no larger than 1; otherwise, it does not make sense for source coding. Moreover, lossless compression can be also regarded as a special case of lossy compression with the coding rate no less than the source entropy and the distortion equal to 0 . Therefore, (2) and (3) can represent both lossy and lossless cases, and lossy compression will reduce to lossless compression by setting the coding rate no less than the source entropy.

After encoding, the outputs $\varphi_{1}\left(x_{1}^{n}\right), \varphi_{2}\left(x_{2}^{n}\right)$ and $\varphi_{\mathrm{H}}\left(y^{n}\right)$ are transmitted to a common receiver. In contrast to distributed compressions in the encoders, the decoder can jointly construct the estimates $\hat{x}_{1}^{n}$ and $\hat{x}_{2}^{n}$ from indices $\varphi_{1}\left(x_{1}^{n}\right)$ and $\varphi_{2}\left(x_{2}^{n}\right)$ by utilizing the compressed side information $\varphi_{\mathrm{H}}\left(y^{n}\right)$. The reconstruction process can be implemented by the mapping as follows:

$$
\psi: \mathcal{M}_{1} \times \mathcal{M}_{2} \times \mathcal{M}_{\mathrm{H}} \mapsto \mathcal{X}_{1}^{n} \times \mathcal{X}_{2}^{n} .
$$

Since the estimate $\hat{x}_{j}^{n}$ may occasionally deviate from the observation $x_{j}^{n}$ if the rates are not large enough, the Hamming distortion measure $d_{j}: \mathcal{X}_{j} \times \mathcal{X}_{j} \mapsto\{0,1\}$ is defined to describe the distortion level between $x_{j}$ and $\hat{x}_{j}$ as

$$
d_{j}\left(x_{j}, \hat{x}_{j}\right)=\left\{\begin{array}{ll}
1, & \text { if } x_{j} \neq \hat{x}_{j}, \\
0, & \text { if } x_{j}=\hat{x}_{j},
\end{array} \text { for } j=1,2 .\right.
$$

Moreover, we define the average distortion between the sequences $x_{j}^{n}$ and $\hat{x}_{j}^{n}$ as

$$
d_{j}\left(x_{j}^{n}, \hat{x}_{j}^{n}\right)=\frac{1}{n} \sum_{i=1}^{n} d_{j}\left(x_{j}(i), \hat{x}_{j}(i)\right), j=1,2 .
$$

\footnotetext{
${ }^{2}$ If the helper sequence is simply generated by concatenating all source sequences together, the helper sequence will have longer sequence length. However, in general, the resources allocated to the helper link are no more than that of the original source link. Therefore, it is reasonable to assume that the helper sequence has the length of $n$ for feasibility and efficiency.
} 
For given distortion requirements $\left(D_{1}, D_{2}\right)$, the rate-distortion region $\mathcal{R}\left(D_{1}, D_{2}\right)$, consisting of all achievable rate triplets $\left(R_{1}, R_{2}, R_{\mathrm{H}}\right)$, is defined as

$$
\begin{aligned}
\mathcal{R}\left(D_{1}, D_{2}\right) & \\
=\left\{\left(R_{1}, R_{2}, R_{\mathrm{H}}\right):\right. & \left(R_{1}, R_{2}, R_{\mathrm{H}}\right) \text { is admissible such that } \\
& \lim _{n \rightarrow \infty} \mathrm{E}\left(d_{j}\left(x_{j}^{n}, \hat{x}_{j}^{n}\right)\right) \leq D_{j}+\epsilon \\
& \text { for } j=1,2, \text { and any } \epsilon>0\} .
\end{aligned}
$$

\section{B. Channel Model}

Due to the independent block Rayleigh fading assumption on each link, the complex channel gains in the link of $X_{1}$, $X_{2}$ and $Y$ are $h_{1}, h_{2}$ and $h_{\mathrm{H}}$, following the two dimensional Gaussian distribution.

It should be emphasized that the step of multiterminal source coding is only utilized to equivalently derive the theoretical performance limit, which can be achieved by the coding scheme used for the achievability proof of Shannon's lossy source-channel separation theorem. However, in the practical system depicted in Fig. 1, the sensor and helper sequences are not actually encoded into the codeword $M$ for lossy compression. As explained above, this difference does not violate the theorem, which provides a universal inner bound. Therefore, in the derivation of outage probability, we use $s$ and $s^{\prime}$ to denote the modulated symbols and the received signals, respectively. The signals received at the $i$-th time index are expressed as

$$
\begin{aligned}
s_{j}^{\prime}(i) & =\sqrt{G_{j}} h_{j} s_{j}(i)+z_{j}(i), \text { for } j=1,2, \\
s_{\mathrm{H}}^{\prime}(i) & =\sqrt{G_{\mathrm{H}}} h_{\mathrm{H}} s_{\mathrm{H}}(i)+z_{\mathrm{H}}(i),
\end{aligned}
$$

where $G$ is the geometric gain, and $z$ represents the zero-mean additive white Gaussian noise (AWGN).

Let $E_{j}=\mathrm{E}\left[\left|s_{j}(i)\right|^{2}\right]$ and $E_{\mathrm{H}}=\mathrm{E}\left[\left|s_{\mathrm{H}}(i)\right|^{2}\right]$ be the transmitting symbol energies, and the variance of all $z$ be equal to $N_{0} / 2$ per dimension. The average SNRs are given by

$$
\begin{aligned}
& \bar{\gamma}_{j}=G_{j} \cdot \mathrm{E}\left[\left|h_{j}\right|^{2}\right] \cdot \frac{E_{j}}{N_{0}}, \text { for } j=1,2, \\
& \bar{\gamma}_{\mathrm{H}}=G_{\mathrm{H}} \cdot \mathrm{E}\left[\left|h_{\mathrm{H}}\right|^{2}\right] \cdot \frac{E_{\mathrm{H}}}{N_{0}} .
\end{aligned}
$$

Then, the instantaneous SNRs can be calculated by

$$
\begin{aligned}
\gamma_{j} & =\left|h_{j}\right|^{2} \cdot \bar{\gamma}_{j}, \text { for } j=1,2, \\
\gamma_{\mathbf{H}} & =\left|h_{\mathbf{H}}\right|^{2} \cdot \bar{\gamma}_{\mathbf{H}} .
\end{aligned}
$$

We can finally obtain the probability density functions (PDFs) of instantaneous SNR as

$$
\begin{aligned}
& p\left(\gamma_{j}\right)=\frac{1}{\bar{\gamma}_{j}} \exp \left(-\frac{\gamma_{j}}{\bar{\gamma}_{j}}\right), \text { for } j=1,2, \\
& p\left(\gamma_{\mathrm{H}}\right)=\frac{1}{\bar{\gamma}_{\mathrm{H}}} \exp \left(-\frac{\gamma_{\mathrm{H}}}{\bar{\gamma}_{\mathrm{H}}}\right) .
\end{aligned}
$$

\section{Rate-Distortion Analysis}

\section{A. Achievable Rate-Distortion Region}

First, from [27], the inner bound on the achievable ratedistortion region with general sources is

$$
R_{1}>I\left(X_{1} ; U_{1} \mid U_{2}, V, Q\right),
$$

$$
\begin{aligned}
R_{2} & >I\left(X_{2} ; U_{2} \mid U_{1}, V, Q\right), \\
R_{1}+R_{2} & >I\left(X_{1}, X_{2} ; U_{1}, U_{2} \mid V, Q\right), \\
R_{\mathrm{H}} & >I(Y ; V),
\end{aligned}
$$

where $U_{j}$ and $V$ are auxiliary variables containing the compressed information in $M_{j}$ and $M_{\mathrm{H}}$ for $X_{j}$ and $Y$, respectively; $Q$ is an auxiliary variable resulting from time-sharing [28] between the cases that one of the coding rates is large enough to independently satisfy the corresponding distortion requirement. Since $Q$ is an auxiliary variable of time-sharing, we calculate the inner bound with binary sources for $|\mathcal{Q}|=1$ for the first step. Then, we equivalently implement the timesharing scheme by using a dummy variable. Consider

$$
\begin{aligned}
R_{1}> & I\left(X_{1} ; U_{1} \mid U_{2}, V\right) \\
= & H\left(U_{1} \mid U_{2}, V\right)-H\left(U_{1} \mid X_{1}, U_{2}, V\right) \\
= & H\left(U_{1} \mid U_{2}, V\right)-H\left(U_{1} \mid X_{1}, X_{2}, U_{2}, V\right) \\
& -I\left(U_{1} ; X_{2} \mid X_{1}, U_{2}, V\right) \\
= & H\left(U_{1} \mid U_{2}, V\right)-H\left(U_{1} \mid X_{1}, X_{2}, U_{2}\right) \\
& -I\left(U_{1} ; X_{2} \mid X_{1}, U_{2}, V\right) \\
= & H\left(U_{1} \mid U_{2}, V\right)-H\left(U_{1} \mid X_{1}\right) \\
& -I\left(U_{1} ; X_{2} \mid X_{1}, U_{2}, V\right) \\
= & H\left(U_{1} \mid U_{2}, V\right)-H\left(U_{1} \mid X_{1}\right)-H\left(X_{2} \mid X_{1}, U_{2}, V\right) \\
& +H\left(X_{2} \mid X_{1}, U_{1}, U_{2}, V\right) \\
= & H\left(U_{1} \mid U_{2}, V\right)-H\left(U_{1} \mid X_{1}\right)-H\left(X_{2} \mid X_{1}, U_{2}, V\right) \\
& +H\left(X_{2} \mid X_{1}, U_{2}, V\right) \\
= & H\left(U_{1} \mid U_{2}, V\right)-H\left(U_{1} \mid X_{1}\right) \\
= & H\left(U_{1} \mid U_{2}\right)-I\left(U_{1} ; V \mid U_{2}\right)-H\left(U_{1} \mid X_{1}\right) \\
= & H_{2}\left(D_{1} * \rho * D_{2}\right)-I\left(U_{1} ; V \mid U_{2}\right)-H_{2}\left(D_{1}\right)
\end{aligned}
$$

where $H_{2}(\cdot)$ is the binary entropy function and the operation $*$ denotes the binary convolution process, i.e., $a * b=a(1-$ b) $+b(1-a)$. (20-22) follow since $V \rightarrow Y \rightarrow\left(X_{1}, X_{2}\right) \rightarrow$ $U_{1}, U_{2} \rightarrow X_{2} \rightarrow X_{1} \rightarrow U_{1}$ and $U_{1} \rightarrow X_{1} \rightarrow X_{2}$ form three Markov chains, respectively, with the first Markov chain resulting from the fact that $Y$ is a function of $X_{1}$ and $X_{2}$. Symmetrically, we have

$$
R_{2}>H_{2}\left(D_{1} * \rho * D_{2}\right)-H_{2}\left(D_{2}\right)-I\left(U_{2} ; V \mid U_{1}\right) .
$$

Then, consider

$$
\begin{aligned}
R_{1}+R_{2}> & I\left(X_{1}, X_{2} ; U_{1}, U_{2} \mid V\right) \\
= & H\left(U_{1}, U_{2} \mid V\right)-H\left(U_{1}, U_{2} \mid X_{1}, X_{2}, V\right) \\
= & H\left(U_{1}, U_{2} \mid V\right)-H\left(U_{1}, U_{2} \mid X_{1}, X_{2}\right) \\
= & H\left(U_{1}, U_{2}\right)-I\left(U_{1}, U_{2} ; V\right)-H\left(U_{1}, U_{2} \mid X_{1}, X_{2}\right) \\
= & H\left(U_{1}\right)+H\left(U_{2} \mid U_{1}\right)-I\left(U_{1}, U_{2} ; V\right) \\
& -H\left(U_{1} \mid X_{1}, X_{2}\right)-H\left(U_{2} \mid X_{1}, X_{2}, U_{1}\right) \\
= & H\left(U_{1}\right)+H\left(U_{2} \mid U_{1}\right)-I\left(U_{1}, U_{2} ; V\right) \\
& -H\left(U_{1} \mid X_{1}\right)-H\left(U_{2} \mid X_{2}\right) \\
= & 1+H_{2}\left(D_{1} * \rho * D_{2}\right)-I\left(U_{1}, U_{2} ; V\right)-H_{2}\left(D_{1}\right) \\
& -H_{2}\left(D_{2}\right),
\end{aligned}
$$

where (25) follows since $V \rightarrow Y \rightarrow\left(X_{1}, X_{2}\right) \rightarrow\left(U_{1}, U_{2}\right)$ form a Markov chain, and (26) follows since $X_{2} \rightarrow X_{1} \rightarrow U_{1}$ and $U_{1} \rightarrow X_{1} \rightarrow X_{2} \rightarrow U_{2}$ form two Markov chains. 
Notice that in (23), (24) and (27), it is hard to calculate $I\left(U_{1} ; V \mid U_{2}\right), I\left(U_{2} ; V \mid U_{1}\right)$ and $I\left(U_{1}, U_{2} ; V\right)$ without a specific helper structure. We consider the theoretical optimal case in which the helper sequence contains the mutual information of sources as much as possible. From the Markov chain $U_{1} \rightarrow$ $\left(X_{1}, X_{2}\right) \rightarrow Y \rightarrow V$, we have

$$
\begin{aligned}
I\left(U_{1} ; V \mid U_{2}\right) & \leq I\left(U_{1} ; V\right) \\
& \leq I(Y ; V) \\
& \leq\left[R_{\mathrm{H}}\right]^{-},
\end{aligned}
$$

where $\left[R_{\mathrm{H}}\right]^{-}=\min \left\{1, R_{\mathrm{H}}\right\}$, and hence

$$
R_{1}>H_{2}\left(D_{1} * \rho * D_{2}\right)-H_{2}\left(D_{1}\right)-\left[R_{\mathrm{H}}\right]^{-} .
$$

Likewise, we have

$$
R_{2}>H_{2}\left(D_{1} * \rho * D_{2}\right)-H_{2}\left(D_{2}\right)-\left[R_{\mathrm{H}}\right]^{-} .
$$

Form the Markov chain $\left(U_{1}, U_{2}\right) \rightarrow\left(X_{1}, X_{2}\right) \rightarrow Y \rightarrow V$, we have

$$
\begin{aligned}
I\left(U_{1}, U_{2} ; V\right) & \leq I(Y ; V) \\
& \leq\left[R_{\mathrm{H}}\right]^{-},
\end{aligned}
$$

and

$$
\begin{aligned}
R_{1}+R_{2}> & 1+H_{2}\left(D_{1} * \rho * D_{2}\right)-H_{2}\left(D_{1}\right) \\
& -H_{2}\left(D_{2}\right)-\left[R_{\mathrm{H}}\right]^{-} .
\end{aligned}
$$

To visually present the inner bound with the time-sharing scheme, the rate-distortion region is divided into three parts, as follows:

(a) for some $0 \leq \tilde{d} \leq D_{2}$,

$$
\left\{\begin{array}{l}
R_{1}>H_{2}\left(D_{1} * \rho * \tilde{d}\right)-H_{2}\left(D_{1}\right)-\left[R_{\mathrm{H}}\right]^{-}, \\
R_{2}>1-H_{2}(\tilde{d})
\end{array}\right.
$$

(b) for some $0 \leq \tilde{d} \leq D_{1}$,

$$
\left\{\begin{array}{l}
R_{1}>1-H_{2}(\tilde{d}), \\
R_{2}>H_{2}\left(\tilde{d} * \rho * D_{2}\right)-H_{2}\left(D_{2}\right)-\left[R_{\mathrm{H}}\right]^{-},
\end{array}\right.
$$

(c) common case,

$$
\begin{aligned}
R_{1}+R_{2}> & 1+H_{2}\left(D_{1} * \rho * D_{2}\right)-H_{2}\left(D_{1}\right) \\
& -H_{2}\left(D_{2}\right)-\left[R_{\mathrm{H}}\right]^{-}
\end{aligned}
$$

where $\tilde{d}$ is a dummy variable. We calculate the rates $R_{1}, R_{2}$ and $R_{1}+R_{2}$ with given $D_{1}$ and $D_{2}$, respectively. Then, we plot the rate-distortion region by combining the three parts shown above, which is equivalent to the time-sharing concept.

\section{B. Performance Evaluation}

An overall view of the inner bound on the achievable ratedistortion region is provided in Fig. 3, where the correlation parameter $\rho$ is set at 0.15 and the desired distortion pair $\left(D_{1}, D_{2}\right)$ is set at $(0.05,0.05)$. Clearly, the helper can enhance the robustness of transmissions by expanding the achievable rate-distortion region as $R_{\mathrm{H}}$ increases. However, the above part of the region, i.e., $R_{\mathrm{H}} \geq 1$, does not change even if the helper rate increases.

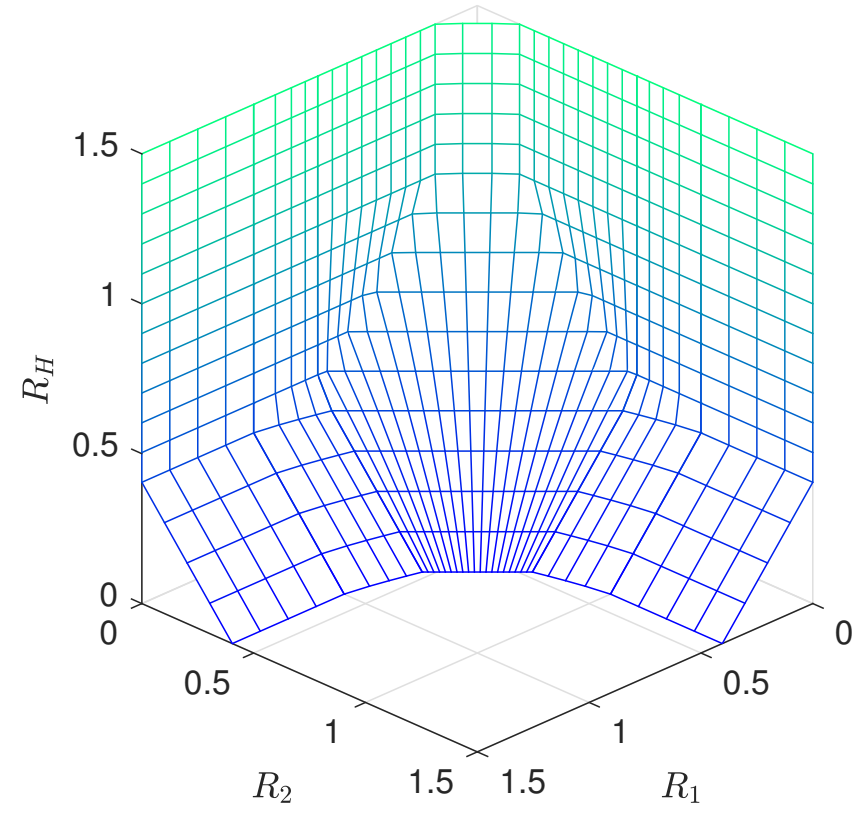

Fig. 3. An overall view of the inner bound on the achievable rate-distortion region, with $\rho=0.15$ and $D_{1}=D_{2}=0.05$.

In order to verify the results by classical theorems, i.e., the Slepian-Wolf theorem and Berger-Tung inner bound, we plot the achievable rate-distortion region for given values of $R_{\mathrm{H}}$ as in Fig. 4. Note that the Slepian-Wolf theorem is for lossless multiterminal source coding, i.e., $D_{1}=D_{2}=0$. Hence, the inner bound is closer to the Slepian-Wolf region when the required distortions are smaller, as shown in Fig. 4(c); conversely, the constraints on the coding rates become less strict if more distortions are acceptable. Besides, the inner bound derived in this paper perfectly coincides with the BergerTung inner bound when $R_{\mathrm{H}}=0$, in spite of the distortion requirements and the correlation level between sources. This phenomenon results from the fact that the system model shown in Fig. 2 reduces to the model used in the Berger-Tung inner bound, if the helper link is removed, i.e., equivalently $R_{\mathrm{H}}=0$. Consequently, the Berger-Tung inner bound can be utilized as a baseline for comparison when analyzing the performance improvement provided by the helper. It is noticeable that the achievable rate-distortion region is obviously enlarged as the helper rate increases, i.e., the communications become more reliable after introducing a helper.

We can investigate the trade-off between the coding rates and the desired distortions in depth from the curve of the ratedistortion, by setting two source links as symmetric links, i.e., $R_{1}=R_{2}$ and $D_{1}=D_{2}$. As depicted in Fig. 5, the derived inner bound with $R_{\mathrm{H}}=0$ also precisely matches the BergerTung inner bound in terms of the rate-distortion function. For $R_{\mathrm{H}}>0$, we can observe significant performance gain provided by a helper. Another interesting observation is that there are still some distortions when $R_{1}=R_{2}=0$, even if $R_{\mathrm{H}} \geq 1$. The reason for the unavoidable distortion is that $H\left(X_{1}^{n}, X_{2}^{n}\right)=$ $H\left(X_{1}^{n}\right)+H\left(X_{2}^{n} \mid X_{1}^{n}\right)=n+n H_{2}(\rho)>n \geq H\left(Y^{n}\right)$ for all $\rho>0$. Hence, the decoder can losslessly reconstruct $X_{1}$ and $X_{2}$ only by the helper information $Y$, if and only if the 


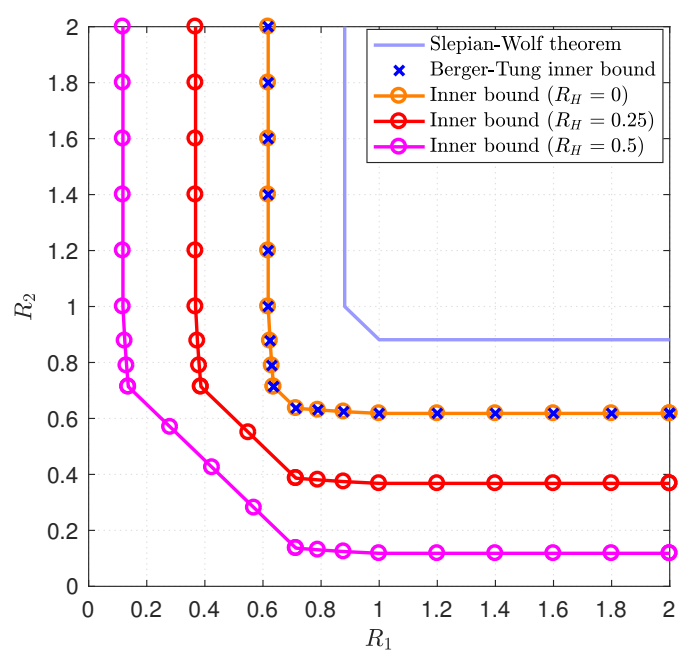

(a) $\rho=0.3, D_{1}=D_{2}=0.05$.

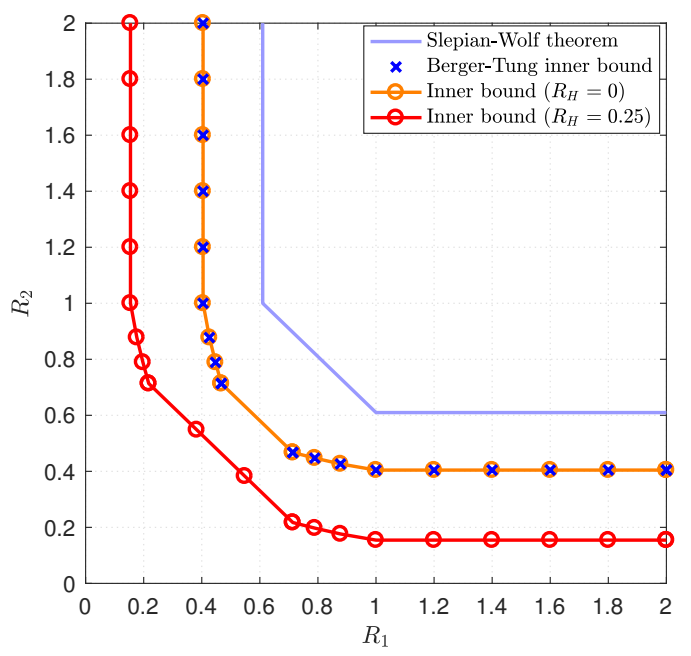

(b) $\rho=0.15, D_{1}=D_{2}=0.05$.

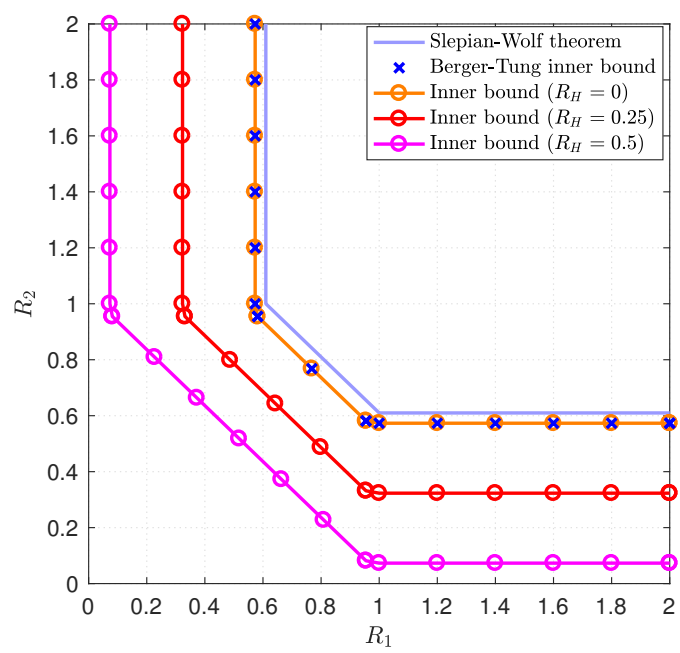

(c) $\rho=0.15, D_{1}=D_{2}=0.005$.

Fig. 4. The achievable rate-distortion region for given $R_{\mathrm{H}}$.

sources are completely correlated. There is also no doubt that

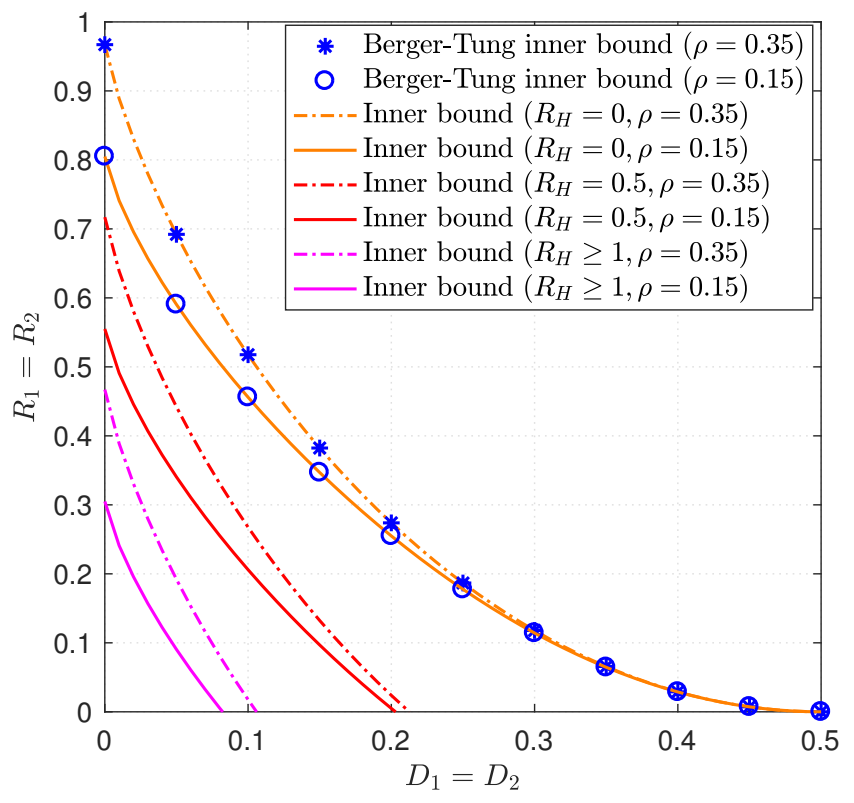

Fig. 5. The rate-distortion function for symmetric source links.

more correlated sources, i.e., $\rho$ is smaller, require lower coding rates for satisfying the same distortion requirements. However, the gap between the curves with different $\rho$ becomes narrower as the distortion requirements go larger. Therefore, with the increment of the desired distortions, the correlations between sources have less effect on the coding rates.

\section{Outage Probability Analysis}

\section{A. Derivation of Outage Probability}

From Shannon's lossy source-channel separation theorem, the distortion requirements $\left(D_{1}, D_{2}\right)$ can be satisfied if the following inequalities hold:

$$
\begin{aligned}
R_{j}\left(D_{j}\right) & \leq \Theta_{j}\left(\gamma_{j}\right)=\frac{C\left(\gamma_{j}\right)}{r_{j}}, \text { for } j=1,2, \\
R_{\mathrm{H}} & \leq \Theta_{\mathrm{H}}\left(\gamma_{\mathrm{H}}\right)=\frac{C\left(\gamma_{\mathrm{H}}\right)}{r_{\mathrm{H}}},
\end{aligned}
$$

where $C(\cdot)$ is the Shannon capacity using Gaussian codebook; $r_{j}$ and $r_{\mathrm{H}}$ stand for the end-to-end coding rates. Therefore, if the link rates $\left(R_{1}, R_{2}, R_{\mathrm{H}}\right)$ supported by $\left(\Theta_{1}\left(\gamma_{1}\right), \Theta_{2}\left(\gamma_{2}\right), \Theta_{\mathrm{H}}\left(\gamma_{\mathrm{H}}\right)\right)$ fall outside the achievable ratedistortion region, the distortion requirements $\left(D_{1}, D_{2}\right)$ cannot be satisfied, i.e., outage event occurs.

For a given value of $R_{\mathrm{H}}$ and specified distortion requirements $\left(D_{1}, D_{2}\right)$, the shape of achievable rate-distortion region can be illustrated as Fig. 6. In order to calculate the outage probability, we divide the area of outage into six subareas with corresponding probabilities $P_{1}, P_{2}, \cdots, P_{6}$. Hence, the outage probability is calculated as

$$
P_{\text {out }}=\sum_{k=1}^{6} P_{k} .
$$




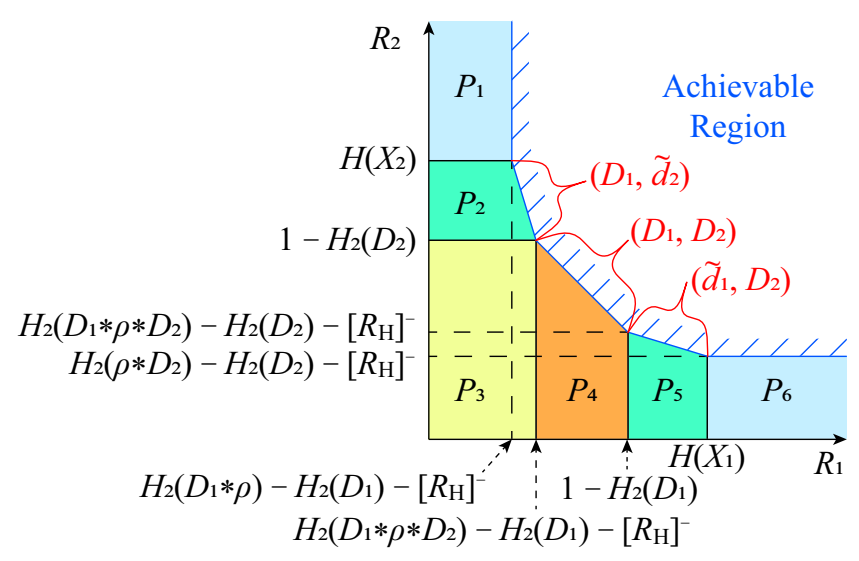

Fig. 6. Achievable rate-distortion region for calculating outage probability.

With specified distortion requirements, the probabilities $P_{1}, P_{2}, \cdots, P_{6}$ are directly determined by the multiple integral with respect to the link rates $\left(R_{1}, R_{2}, R_{\mathrm{H}}\right)$ for each subarea. Since the link rates are eventually supported by the instantaneous SNRs, the probabilities $P_{1}, P_{2}, \cdots, P_{6}$ can be finally calculated by the multiple integral of the joint PDF with respect to the instantaneous SNRs.

First, consider

$$
\begin{gathered}
P_{1}=\operatorname{Pr}\left\{0 \leq R_{1} \leq H_{2}\left(D_{1} * \rho\right)-H_{2}\left(D_{1}\right)-\left[R_{\mathrm{H}}\right]^{-},\right. \\
\left.H\left(X_{2}\right) \leq R_{2}, 0 \leq R_{\mathrm{H}}\right\} \\
=\operatorname{Pr}\left\{0 \leq \Theta_{1}\left(\gamma_{1}\right) \leq \lambda_{1}(0), H\left(X_{2}\right) \leq \Theta_{2}\left(\gamma_{2}\right),\right. \\
\left.0 \leq \Theta_{\mathrm{H}}\left(\gamma_{\mathrm{H}}\right)\right\} \\
=\operatorname{Pr}\left\{\Theta_{1}^{-1}(0) \leq \gamma_{1} \leq \Theta_{1}^{-1}\left[\lambda_{1}(0)\right],\right. \\
\left.\Theta_{2}^{-1}\left[H\left(X_{2}\right)\right] \leq \gamma_{2}, \Theta_{\mathrm{H}}^{-1}(0) \leq \gamma_{\mathrm{H}}\right\} \\
=\int_{\Theta_{\mathrm{H}}^{-1}(0)}^{\infty} d \gamma_{\mathrm{H}} \int_{\Theta_{1}^{-1}(0)}^{\Theta_{1}^{-1}\left[\lambda_{1}(0)\right]} d \gamma_{1} \\
\cdot \int_{\Theta_{2}^{-1}\left[H\left(X_{2}\right)\right]}^{\infty} p\left(\gamma_{2}\right) p\left(\gamma_{1}\right) p\left(\gamma_{\mathrm{H}}\right) d \gamma_{2},
\end{gathered}
$$

where $\lambda_{j}(\tilde{d})=\max \left\{0, H_{2}\left(D_{j} * \rho * \tilde{d}\right)-H_{2}\left(D_{j}\right)-\left[\Theta_{\mathrm{H}}\left(\gamma_{\mathrm{H}}\right)\right]^{-}\right\}$ for $j=1,2$. Notice that $H_{2}\left(D_{j} * \rho * \tilde{d}\right) \leq 1$ and $H_{2}\left(D_{j}\right) \geq 0$; hence, $\lambda_{j}(\tilde{d})=0$ when $\Theta_{\mathrm{H}}\left(\gamma_{\mathrm{H}}\right)=R_{\mathrm{H}}>1$. Therefore, $P_{1}$ can be further calculated as

$$
\begin{aligned}
P_{1}= & \int_{\Theta_{\mathrm{H}}^{-1}(0)}^{\Theta_{\mathrm{H}}^{-1}(1)} d \gamma_{\mathrm{H}} \int_{\Theta_{1}^{-1}(0)}^{\Theta_{1}^{-1}\left[\lambda_{1}(0)\right]} d \gamma_{1} \\
& \cdot \int_{\Theta_{2}^{-1}\left[H\left(X_{2}\right)\right]}^{\infty} p\left(\gamma_{2}\right) p\left(\gamma_{1}\right) p\left(\gamma_{\mathrm{H}}\right) d \gamma_{2} \\
& +\int_{\Theta_{\mathrm{H}}^{-1}(1)}^{\infty} d \gamma_{\mathrm{H}} \int_{\Theta_{1}^{-1}(0)}^{\Theta_{1}^{-1}[0]} d \gamma_{1} \\
& \cdot \int_{\Theta_{2}^{-1}\left[H\left(X_{2}\right)\right]}^{\infty} p\left(\gamma_{2}\right) p\left(\gamma_{1}\right) p\left(\gamma_{\mathrm{H}}\right) d \gamma_{2} \\
= & \int_{\Theta_{\mathrm{H}}^{-1}(0)}^{\Theta_{\mathrm{H}}^{-1}(1)} d \gamma_{\mathrm{H}} \int_{\Theta_{1}^{-1}(0)}^{\Theta_{1}^{-1}\left[\lambda_{1}(0)\right]} d \gamma_{1} \\
& \cdot \int_{\Theta_{2}^{-1}\left[H\left(X_{2}\right)\right]}^{\infty} p\left(\gamma_{2}\right) p\left(\gamma_{1}\right) p\left(\gamma_{\mathrm{H}}\right) d \gamma_{2}+0
\end{aligned}
$$

$$
\begin{aligned}
= & \frac{1}{\bar{\gamma}_{\mathrm{H}}} \cdot \exp \left(-\frac{\Theta_{2}^{-1}\left[H\left(X_{2}\right)\right]}{\bar{\gamma}_{2}}\right) \cdot \int_{\Theta_{\mathrm{H}}^{-1}(0)}^{\Theta_{\mathrm{H}}^{-1}(1)} \exp \left(-\frac{\gamma_{\mathrm{H}}}{\bar{\gamma}_{\mathrm{H}}}\right) \\
& \cdot\left[1-\exp \left(-\frac{\Theta_{1}^{-1}\left[\lambda_{1}(0)\right]}{\bar{\gamma}_{1}}\right)\right] d \gamma_{\mathrm{H}} .
\end{aligned}
$$

Next, consider

$$
\begin{aligned}
& P_{2}=\operatorname{Pr}\left\{0 \leq R_{1} \leq H_{2}\left(D_{1} * \rho * \tilde{d}_{2}\right)-H_{2}\left(D_{1}\right)-\left[R_{\mathrm{H}}\right]^{-},\right. \\
& \left.1-H_{2}\left(D_{2}\right) \leq R_{2}<H\left(X_{2}\right), 0 \leq R_{\mathrm{H}}\right\} \\
& =\operatorname{Pr}\left\{\Theta_{1}^{-1}(0) \leq \gamma_{1} \leq \Theta_{1}^{-1}\left[\lambda_{1}\left(\tilde{d}_{2}\right)\right]\right. \text {, } \\
& \Theta_{2}^{-1}\left[1-H_{2}\left(D_{2}\right)\right] \leq \gamma_{2}<\Theta_{2}^{-1}\left[H\left(X_{2}\right)\right], \\
& \left.\Theta_{\mathrm{H}}^{-1}(0) \leq \gamma_{\mathrm{H}}\right\} \\
& =\int_{\Theta_{\mathrm{H}}^{-1}(0)}^{\infty} d \gamma_{\mathrm{H}} \int_{\Theta_{2}^{-1}\left[1-H_{2}\left(D_{2}\right)\right]}^{\Theta_{2}^{-1}\left[H\left(X_{2}\right)\right]} d \gamma_{2} \\
& \cdot \int_{\Theta_{1}^{-1}(0)}^{\Theta_{1}^{-1}\left[\lambda_{1}\left(\tilde{d}_{2}\right)\right]} p\left(\gamma_{1}\right) p\left(\gamma_{2}\right) p\left(\gamma_{\mathbf{H}}\right) d \gamma_{1} \\
& =\int_{\Theta_{\mathrm{H}}^{-1}(0)}^{\Theta_{\mathrm{H}}^{-1}(1)} d \gamma_{\mathrm{H}} \int_{\Theta_{2}^{-1}\left[1-H_{2}\left(D_{2}\right)\right]}^{\Theta_{2}^{-1}\left[H\left(X_{2}\right)\right]} d \gamma_{2} \\
& \cdot \int_{\Theta_{1}^{-1}(0)}^{\Theta_{1}^{-1}\left[\lambda_{1}\left(\tilde{d}_{2}\right)\right]} p\left(\gamma_{1}\right) p\left(\gamma_{2}\right) p\left(\gamma_{\mathbf{H}}\right) d \gamma_{1} \\
& +\int_{\Theta_{\mathrm{H}}^{-1}(1)}^{\infty} d \gamma_{\mathrm{H}} \int_{\Theta_{2}^{-1}\left[1-H_{2}\left(D_{2}\right)\right]}^{\Theta_{2}^{-1}\left[H\left(X_{2}\right)\right]} d \gamma_{2} \\
& \cdot \int_{\Theta_{1}^{-1}(0)}^{\Theta_{1}^{-1}[0]} p\left(\gamma_{1}\right) p\left(\gamma_{2}\right) p\left(\gamma_{\mathbf{H}}\right) d \gamma_{1} \\
& =\int_{\Theta_{\mathrm{H}}^{-1}(0)}^{\Theta_{\mathrm{H}}^{-1}(1)} d \gamma_{\mathrm{H}} \int_{\Theta_{2}^{-1}\left[1-H_{2}\left(D_{2}\right)\right]}^{\Theta_{2}^{-1}\left[H\left(X_{2}\right)\right]} d \gamma_{2} \\
& \cdot \int_{\Theta_{1}^{-1}(0)}^{\Theta_{1}^{-1}\left[\lambda_{1}\left(\tilde{d}_{2}\right)\right]} p\left(\gamma_{1}\right) p\left(\gamma_{2}\right) p\left(\gamma_{\mathrm{H}}\right) d \gamma_{1}+0 \\
& =\frac{1}{\bar{\gamma}_{2} \bar{\gamma}_{\mathrm{H}}} \cdot \int_{\Theta_{\mathrm{H}}^{-1}(0)}^{\Theta_{\mathrm{H}}^{-1}(1)} d \gamma_{\mathrm{H}} \\
& \cdot \int_{\Theta_{2}^{-1}\left[1-H_{2}\left(D_{2}\right)\right]}^{\Theta_{2}^{-1}\left[H\left(X_{2}\right)\right]} \exp \left(-\frac{\gamma_{2}}{\bar{\gamma}_{2}}-\frac{\gamma_{\mathrm{H}}}{\bar{\gamma}_{\mathrm{H}}}\right) \\
& \cdot\left[1-\exp \left(-\frac{\Theta_{1}^{-1}\left[\lambda_{1}\left(\tilde{d}_{2}\right)\right]}{\bar{\gamma}_{1}}\right)\right] d \gamma_{2} \text {, }
\end{aligned}
$$

where $\tilde{d}_{j}=H_{2}^{-1}\left(1-\left[R_{j}\right]^{-}\right)$for $j=1,2$.

For $P_{3}$, consider

$$
\begin{gathered}
P_{3}=\operatorname{Pr}\left\{0 \leq R_{1}<H_{2}\left(D_{1} * \rho * D_{2}\right)-H_{2}\left(D_{1}\right)-\left[R_{\mathrm{H}}\right]^{-},\right. \\
\left.0 \leq R_{2}<1-H_{2}\left(D_{2}\right), 0 \leq R_{\mathrm{H}}\right\} \\
=\operatorname{Pr}\left\{\Theta_{1}^{-1}(0) \leq \gamma_{1}<\Theta_{1}^{-1}\left[\lambda_{1}\left(D_{2}\right)\right]\right. \\
\Theta_{2}^{-1}(0) \leq \gamma_{2}<\Theta_{2}^{-1}\left[1-H_{2}\left(D_{2}\right)\right] \\
\left.\Theta_{\mathrm{H}}^{-1}(0) \leq \gamma_{\mathrm{H}}\right\} \\
=\int_{\Theta_{\mathrm{H}}^{-1}(0)}^{\infty} d \gamma_{\mathrm{H}} \int_{\Theta_{2}^{-1}(0)}^{\Theta_{2}^{-1}\left[1-H_{2}\left(D_{2}\right)\right]} d \gamma_{2} \\
\cdot \int_{\Theta_{1}^{-1}(0)}^{\Theta_{1}^{-1}\left[\lambda_{1}\left(D_{2}\right)\right]} p\left(\gamma_{1}\right) p\left(\gamma_{2}\right) p\left(\gamma_{\mathrm{H}}\right) d \gamma_{1}
\end{gathered}
$$




$$
\begin{aligned}
= & \int_{\Theta_{\mathrm{H}}^{-1}(0)}^{\Theta_{\mathrm{H}}^{-1}(1)} d \gamma_{\mathrm{H}} \int_{\Theta_{2}^{-1}(0)}^{\Theta_{2}^{-1}\left[1-H_{2}\left(D_{2}\right)\right]} d \gamma_{2} \\
& \cdot \int_{\Theta_{1}^{-1}(0)}^{\Theta_{1}^{-1}\left[\lambda_{1}\left(D_{2}\right)\right]} p\left(\gamma_{1}\right) p\left(\gamma_{2}\right) p\left(\gamma_{\mathrm{H}}\right) d \gamma_{1} \\
& +\int_{\Theta_{\mathrm{H}}^{-1}(1)}^{\infty} d \gamma_{\mathrm{H}} \int_{\Theta_{2}^{-1}(0)}^{\Theta_{2}^{-1}\left[1-H_{2}\left(D_{2}\right)\right]} d \gamma_{2} \\
& \cdot \int_{\Theta_{1}^{-1}(0)}^{\Theta_{1}^{-1}[0]} p\left(\gamma_{1}\right) p\left(\gamma_{2}\right) p\left(\gamma_{\mathrm{H}}\right) d \gamma_{1} \\
= & \int_{\Theta_{\mathrm{H}}^{-1}(0)}^{\Theta_{\mathrm{H}}^{-1}(1)} d \gamma_{\mathrm{H}} \int_{\Theta_{2}^{-1}(0)}^{\Theta_{2}^{-1}\left[1-H_{2}\left(D_{2}\right)\right]} d \gamma_{2} \\
& \cdot \int_{\Theta_{1}^{-1}(0)}^{\Theta_{1}^{-1}\left[\lambda_{1}\left(D_{2}\right)\right]} p\left(\gamma_{1}\right) p\left(\gamma_{2}\right) p\left(\gamma_{\mathrm{H}}\right) d \gamma_{1}+0 \\
= & \frac{1}{\bar{\gamma}_{\mathrm{H}}} \cdot\left[1-\exp \left(-\frac{\Theta_{2}^{-1}\left[1-H_{2}\left(D_{2}\right)\right]}{\bar{\gamma}_{2}}\right)\right] \\
& \cdot \int_{\Theta_{\mathrm{H}}^{-1}(0)}^{\Theta_{\mathrm{H}}^{-1}(1)} \exp \left(-\frac{\gamma_{\mathrm{H}}}{\bar{\gamma}_{\mathrm{H}}}\right) \\
& \cdot\left[1-\exp \left(-\frac{\Theta_{1}^{-1}\left[\lambda_{1}\left(D_{2}\right)\right]}{\bar{\gamma}_{1}}\right)\right] d \gamma_{\mathrm{H}} .
\end{aligned}
$$

To calculate $P_{4}$, consider

$$
\begin{aligned}
& P_{4}=\operatorname{Pr}\left\{H_{2}\left(D_{1} * \rho * D_{2}\right)-H_{2}\left(D_{1}\right)-\left[R_{\mathrm{H}}\right]^{-}\right. \\
& \leq R_{1}<1-H_{2}\left(D_{1}\right) \text {, } \\
& 0 \leq R_{2} \leq 1+H_{2}\left(D_{1} * \rho * D_{2}\right)-H_{2}\left(D_{1}\right) \\
& -H_{2}\left(D_{2}\right)-\left[R_{\mathrm{H}}\right]^{-}-\left[R_{1}\right]^{-} \text {, } \\
& \left.0 \leq R_{\mathrm{H}}\right\} \\
& =\operatorname{Pr}\left\{\Theta_{1}^{-1}\left[\lambda_{1}\left(D_{2}\right)\right] \leq \gamma_{1} \leq \Theta_{1}^{-1}\left[1-H_{2}\left(D_{1}\right)\right],\right. \\
& \left.\Theta_{2}^{-1}(0) \leq \gamma_{2} \leq \Theta_{2}^{-1}\left(\mu_{1}\right), \Theta_{\mathrm{H}}^{-1}(0) \leq \gamma_{\mathrm{H}}\right\} \\
& =\int_{\Theta_{\mathrm{H}}^{-1}(0)}^{\infty} d \gamma_{\mathrm{H}} \int_{\Theta_{1}^{-1}\left[\lambda_{1}\left(D_{2}\right)\right]}^{\Theta_{1}^{-1}\left[1-H_{2}\left(D_{1}\right)\right]} d \gamma_{1} \\
& \cdot \int_{\Theta_{2}^{-1}(0)}^{\Theta_{2}^{-1}\left(\mu_{1}\right)} p\left(\gamma_{2}\right) p\left(\gamma_{1}\right) p\left(\gamma_{\mathrm{H}}\right) d \gamma_{2} \\
& =\int_{\Theta_{\mathrm{H}}^{-1}(0)}^{\Theta_{\mathrm{H}}^{-1}(1)} d \gamma_{\mathrm{H}} \int_{\Theta_{1}^{-1}\left[\lambda_{1}\left(D_{2}\right)\right]}^{\Theta_{1}^{-1}\left[1-H_{2}\left(D_{1}\right)\right]} d \gamma_{1} \\
& \cdot \int_{\Theta_{2}^{-1}(0)}^{\Theta_{2}^{-1}\left(\mu_{1}\right)} p\left(\gamma_{2}\right) p\left(\gamma_{1}\right) p\left(\gamma_{\mathrm{H}}\right) d \gamma_{2} \\
& +\int_{\Theta_{\mathrm{H}}^{-1}(1)}^{\infty} d \gamma_{\mathrm{H}} \int_{\Theta_{1}^{-1}[0]}^{\Theta_{1}^{-1}\left[1-H_{2}\left(D_{1}\right)\right]} d \gamma_{1} \\
& \cdot \int_{\Theta_{2}^{-1}(0)}^{\Theta_{2}^{-1}\left(\mu_{1}\right)} p\left(\gamma_{2}\right) p\left(\gamma_{1}\right) p\left(\gamma_{\mathrm{H}}\right) d \gamma_{2} \\
& =\frac{1}{\bar{\gamma}_{1} \bar{\gamma}_{\mathrm{H}}} \cdot \int_{\Theta_{\mathrm{H}}^{-1}(0)}^{\Theta_{\mathrm{H}}^{-1}(1)} d \gamma_{\mathrm{H}} \\
& \cdot \int_{\Theta_{1}^{-1}\left[\lambda_{1}\left(D_{2}\right)\right]}^{\Theta_{1}^{-1}\left[1-H_{2}\left(D_{1}\right)\right]} \exp \left(-\frac{\gamma_{1}}{\bar{\gamma}_{1}}-\frac{\gamma_{\mathrm{H}}}{\bar{\gamma}_{\mathrm{H}}}\right) \\
& \cdot\left[1-\exp \left(-\frac{\Theta_{2}^{-1}\left(\mu_{1}\right)}{\bar{\gamma}_{2}}\right)\right] d \gamma_{1}
\end{aligned}
$$

$$
\begin{aligned}
& +\frac{1}{\bar{\gamma}_{1}} \cdot \exp \left(-\frac{\Theta_{\mathrm{H}}^{-1}(1)}{\bar{\gamma}_{\mathrm{H}}}\right) \\
& \cdot \int_{\Theta_{1}^{-1}[0]}^{\Theta_{1}^{-1}\left[1-H_{2}\left(D_{1}\right)\right]} \exp \left(-\frac{\gamma_{1}}{\bar{\gamma}_{1}}\right) \\
& \cdot\left[1-\exp \left(-\frac{\Theta_{2}^{-1}\left(\mu_{1}^{\prime}\right)}{\bar{\gamma}_{2}}\right)\right] d \gamma_{1},
\end{aligned}
$$

where $\mu_{j}=\max \left\{0,1+H_{2}\left(D_{1} * \rho * D_{2}\right)-H_{2}\left(D_{1}\right)-H_{2}\left(D_{2}\right)-\right.$ $\left.\left[\Theta_{\mathrm{H}}\left(\gamma_{\mathrm{H}}\right)\right]^{-}-\left[\Theta_{j}\left(\gamma_{j}\right)\right]^{-}\right\}$and $\mu_{j}^{\prime}=\max \left\{0, H_{2}\left(D_{1} * \rho * D_{2}\right)-\right.$ $\left.H_{2}\left(D_{1}\right)-H_{2}\left(D_{2}\right)-\left[\Theta_{j}\left(\gamma_{j}\right)\right]^{-}\right\}$for $j=1,2$.

Similar to the calculation of $P_{2}$ and $P_{1}$, we have

$$
\begin{aligned}
P_{5}= & \frac{1}{\bar{\gamma}_{1} \bar{\gamma}_{\mathrm{H}}} \cdot \int_{\Theta_{\mathrm{H}}^{-1}(0)}^{\Theta_{\mathrm{H}}^{-1}(1)} d \gamma_{\mathrm{H}} \\
& \cdot \int_{\Theta_{1}^{-1}\left[1-H_{2}\left(D_{1}\right)\right]}^{\Theta_{1}^{-1}\left[H\left(X_{1}\right)\right]} \exp \left(-\frac{\gamma_{1}}{\bar{\gamma}_{1}}-\frac{\gamma_{\mathrm{H}}}{\bar{\gamma}_{\mathrm{H}}}\right) \\
& \cdot\left[1-\exp \left(-\frac{\Theta_{2}^{-1}\left[\lambda_{2}\left(\tilde{d}_{1}\right)\right]}{\bar{\gamma}_{2}}\right)\right] d \gamma_{1}, \\
P_{6}= & \frac{1}{\bar{\gamma}_{\mathrm{H}}} \cdot \exp \left(-\frac{\Theta_{1}^{-1}\left[H\left(X_{1}\right)\right]}{\bar{\gamma}_{1}}\right) \cdot \int_{\Theta_{\mathrm{H}}^{-1}(0)}^{\Theta_{\mathrm{H}}^{-1}(1)} \exp \left(-\frac{\gamma_{\mathrm{H}}}{\bar{\gamma}_{\mathrm{H}}}\right) \\
& \cdot\left[1-\exp \left(-\frac{\Theta_{2}^{-1}\left[\lambda_{2}(0)\right]}{\bar{\gamma}_{2}}\right)\right] d \gamma_{\mathrm{H}} .
\end{aligned}
$$

Remark: Following the same logic, we can extend the system model to the case with more than two sensors, by applying the achievable rate-distortion region with multiple sources. However, the calculation of outage probability requires more complicated multiple integral.

\section{B. Numerical Results}

The outage probability for lossy communications with a helper is depicted in Fig. 7, where the channel coding rate is set at $\frac{1}{2}$ for all the sensors and the helper. For the purpose of plotting the curves in a 2D plane, the average SNRs are set at different values but change at the same speed for each link, i.e., $\bar{\gamma}_{1}=\bar{\gamma}_{2}+2=\bar{\gamma}_{\mathrm{H}}-3$. It should be explained here that the curves for the case without a helper only show first order diversity, due to the definition of outage event, i.e., the recoveries of the both sources cannot satisfy the distortion requirements. We can clearly observe from the decay of the curves that with a helper, second order diversity can be achieved. Obviously, the case with a helper providing the side information achieves higher diversity order than the case without a helper. It is found from Fig. 7(a) and Fig. 7(b) that the smaller the correlation between the sources, i.e., $\rho$ is larger, the higher the outage probability, which is consistent to our expected results. In addition, it is noticeable that the system will have lower outage probability, if the distortion requirements are less strict. Hence, identifying how to specify the distortion requirements is valuable and interesting work in the design of practical IoT systems.

Fig. 8 illustrates the outage probability for fixed average SNR in the helper link, i.e., only $\bar{\gamma}_{1}=\bar{\gamma}_{2}+2$. It is obvious that the outage curves are shifted to left by increasing the average SNR in the helper link. However, the gap between the 


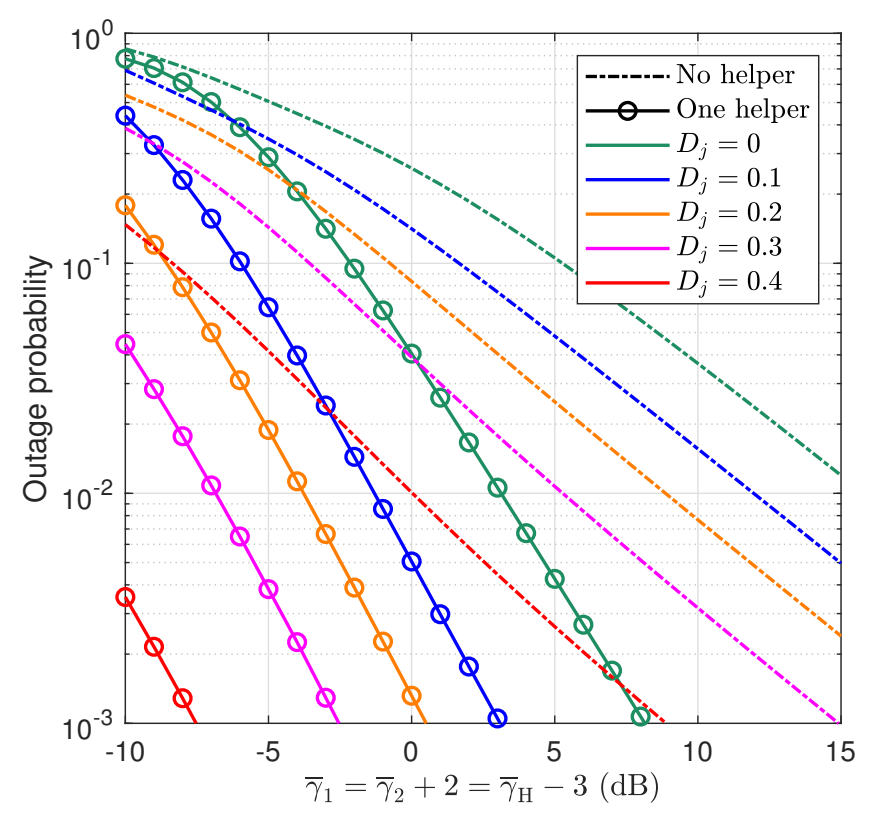

(a) $\rho=0.1$.

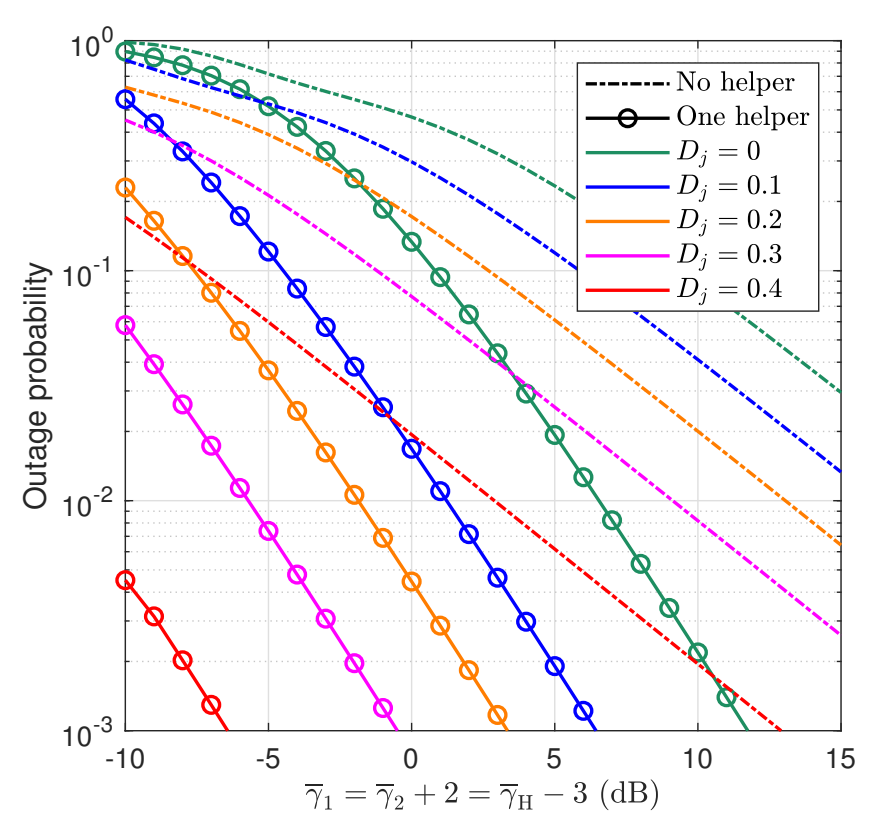

(b) $\rho=0.4$.

Fig. 7. Outage probability for the sources with different correlation levels.

outage curves becomes narrower when $\bar{\gamma}_{\mathrm{H}}$ is relatively small. Because the channel capacity of the helper link cannot support lossless transmission of the helper information if $\gamma_{\mathrm{H}}<0 \mathrm{~dB}$, while there is no loss of the helper information for $\gamma_{\mathrm{H}} \geq 0 \mathrm{~dB}$. Besides, notice that the slope of curve in Fig. 8 is less steep than that of the case with one helper in Fig. 7. The reason for this observation is because the value of $\bar{\gamma}_{\mathrm{H}}$ is fixed (does not change along with $\bar{\gamma}_{1}$ and $\bar{\gamma}_{2}$ ) in Fig. 8, and the effect of performance improvement provided by a helper appears in the form of parallel shifting of outage curves. If we use a dashed line to connect the points where $\bar{\gamma}_{1}=\bar{\gamma}_{2}+2=\bar{\gamma}_{\mathrm{H}}-3$ like in Fig. 7, we can observe second order diversity.

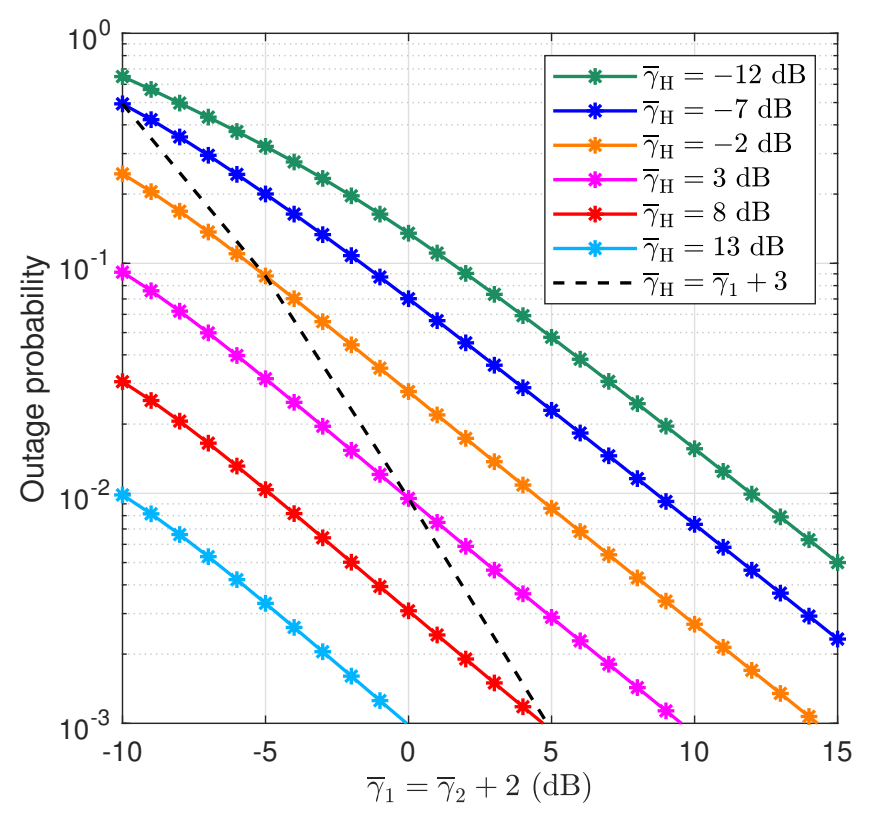

Fig. 8. Outage probability for diverse average SNRs in the helper link, with $\rho=0.2$ and $D_{1}=D_{2}=0.1$.

\section{Practical Performance Evaluation}

\section{A. Simulation Design}

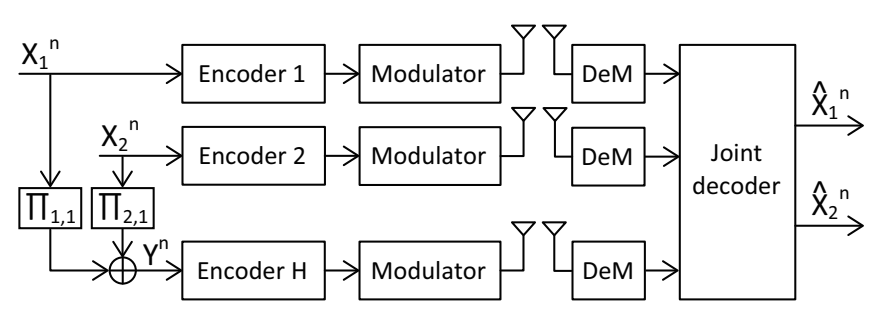

Fig. 9. Simulation system.

In this section, we start to evaluate the outage probability over independent block Rayleigh fading channels for a practical communication system depicted in Fig. 9. Two sensors independently encode two correlated sequences $X_{1}^{n}$ and $X_{2}^{n}$, and then send them to a common receiver after modulation. Meanwhile, a helper generates its information $Y^{n}$ from $X_{1}^{n}$ and $X_{2}^{n}$ by $\mathrm{XOR}^{3}$. Notice that if the helper directly perform $\mathrm{XOR}$ as $Y=X_{1} \oplus X_{2}$ bit by bit, $Y^{n}$ will be almost 0 for highly correlated sources and waste the helper rate. To make the XOR helper more efficient, $X_{1}^{n}$ and $X_{2}^{n}$ are interleaved by two interleavers $\Pi_{1,1}$ and $\Pi_{2,1}$ before XOR, respectively. Subsequently, the helper sequence $Y^{n}$ is encoded, modulated and finally sent to the receiver. At the common receiver, the received signals are jointly decoded after demodulation (DeM), and the joint decoder finally outputs the hard decisions $\hat{X}_{1}^{n}$ and $\hat{X}_{2}^{n}$.

The structure of encoders is illustrated in Fig. 10. Since two sequences $X_{1}^{n}$ and $X_{2}^{n}$ are correlated, $X_{j}^{n}$ is interleaved by

\footnotetext{
${ }^{3}$ Since the optimal helper structure is still an open problem and out of the scope of this paper, we choose a frequently used structure, i.e., XOR, for the practical performance evaluation.
} 


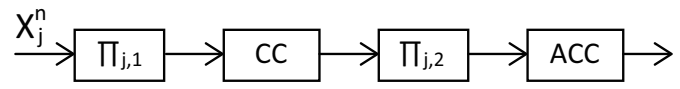

(a) Sensor encoder $j$.

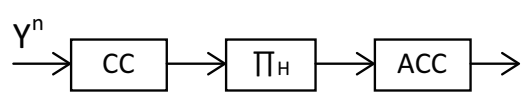

(b) Helper encoder $\mathrm{H}$.

Fig. 10. The structure of encoders.

$\Pi_{j, 1}$ for the first step, so as to disperse noises into different bits. Then, the interleaved version of $X_{j}^{n}$ is successively encoded by a convolutional code (CC) and an accumulator (ACC) [29]. In order to exploit the principle of turbo codes in iterative decoding, another interleaver $\Pi_{j, 2}$ is deployed between $\mathrm{CC}$ and ACC. For the helper encoder, only one interleaver $\Pi_{\mathrm{H}}$ is needed between CC and ACC.

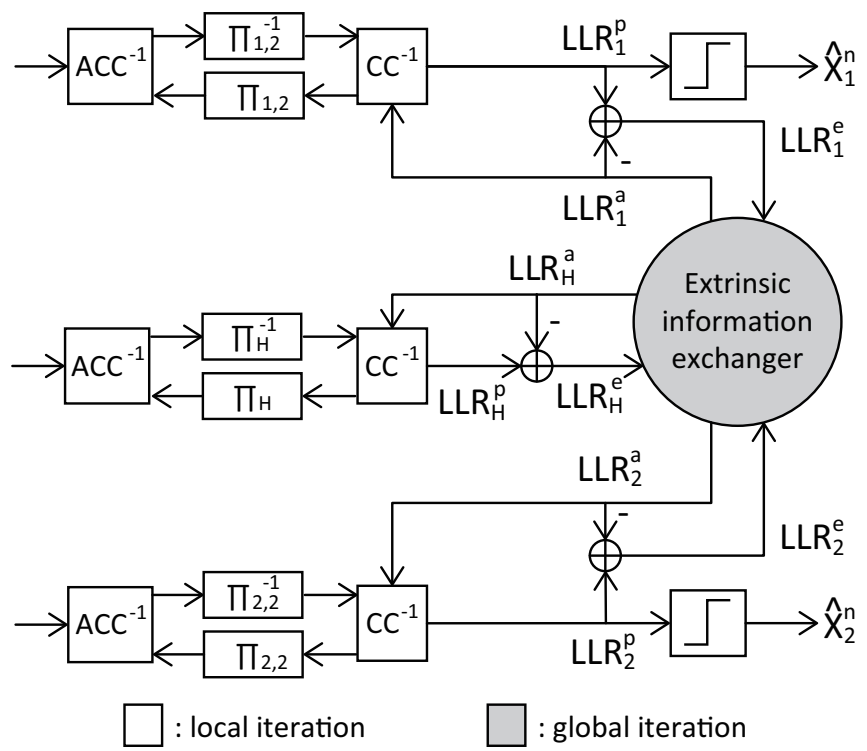

Fig. 11. The structure of joint decoder.

Fig. 11 shows the structure of corresponding joint decoder, which separately decodes the received signals in local iteration and exchanges mutual information in global iteration. In local iteration, the bits encoded by $\mathrm{ACC}$ and $\mathrm{CC}$ are respectively decoded by the corresponding decoders $\mathrm{ACC}^{-1}$ and $\mathrm{CC}^{-1}$, which exchange log-likelihood ratio (LLR) via a deinterleaver $\Pi^{-1}$ and an interleaver $\Pi$. Then, the extrinsic LLR $\left(\operatorname{LLR}^{e}\right)$ utilized in global iteration is calculated by subtracting the a priori LLR $\left(\mathrm{LLR}^{a}\right)$ from the a posteriori $\mathrm{LLR}\left(\mathrm{LLR}^{p}\right)$. Subsequently, the extrinsic information exchanger updates the a priori LLRs based on the extrinsic LLRs yielded from local iteration. The joint decoder performs local iteration and global iteration in turn, until it cannot obtain obvious iteration gains or exceeds the maximum iteration time. Finally, the hard decision $\hat{X}_{j}^{n}$ is made from the a posteriori LLR in the last round of local iteration.

Fig. 12 illustrates the structure of extrinsic information exchanger. Since the helper information is generated by XOR, the extrinsic LLRs are exchanged among two sensors and

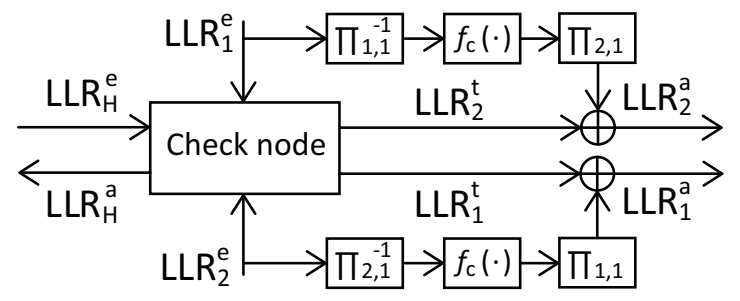

Fig. 12. The structure of extrinsic information exchanger.

the helper, by the same principle of the check node in lowdensity parity-check (LDPC) codes. The check node outputs temporary results $\operatorname{LLR}_{j}^{t}$ for two sensors, and the a priori $\operatorname{LLR}_{\mathrm{H}}^{a}$ for the helper, by

$$
\begin{aligned}
\operatorname{LLR}_{j}^{t}= & -2 \cdot \operatorname{arctanh}\left(\tanh \frac{-\mathrm{LLR}_{k}^{e}}{2} \cdot \tanh \frac{-\mathrm{LLR}_{\mathrm{H}}^{e}}{2}\right) \\
& +\mathrm{LLR}_{j}^{e}, \\
\mathrm{LLR}_{\mathrm{H}}^{a}= & -2 \cdot \operatorname{arctanh}\left(\prod_{j \in\{1,2\}} \tanh \frac{-\mathrm{LLR}_{j}^{e}}{2}\right)+\mathrm{LLR}_{\mathrm{H}}^{e},
\end{aligned}
$$

where $j \in\{1,2\}$ and $k=\{1,2\} \backslash j$. Meanwhile, $X_{1}^{n}$ and $X_{2}^{n}$ also have correlations without the helper. For directly exchanging the extrinsic information between two correlated sources based on the correlation model [30], $\operatorname{LLR}_{j}^{e}$ is processed in the order of $\Pi_{j, 1}^{-1} \rightarrow f_{c}(\cdot) \rightarrow \Pi_{k, 1}$, where $f_{c}(\cdot)$ is the LLR updating function for correlated sources [31]. Finally, the $\operatorname{LLR}_{k}^{a}$ for sensors is calculated by the sum of $\operatorname{LLR}_{k}^{t}$ and the output from $\Pi_{k, 1}$.

\section{B. Simulation Results}

TABLE I

SIMULATION PARAMETERS

\begin{tabular}{c|c}
\hline Parameter & Value \\
\hline \hline Frame length & $10^{4}$ bits \\
\hline Number of frames & $5 \times 10^{5}$ \\
\hline Source coding rate & 1 \\
\hline Channel coding rate & $1 / 2$ \\
\hline Generator polynomial of CC & $G=([3,2] 3)_{8}$ \\
\hline Type of interleaver & random interleaver \\
\hline Modulation method & BPSK \\
\hline Maximum iteration time & 20 \\
\hline
\end{tabular}

With the setting of parameters listed in Table $\mathrm{I}^{4}$, we can obtain the simulation results of frame error rate (FER) with specified distortion requirements as depicted in Fig. 13. For simplicity, the average SNR is set at the same level for all sensor links and the helper link. Clearly, although there is an obvious gap between the simulation and theoretical results, the simulation results show the same tendency as the theoretical results, i.e., second order diversity, and the reduction of outage

\footnotetext{
${ }^{4}$ Due to the long frame length and large number of frames, the simulation requires quite a long time. Moreover, the main purpose of simulations in this paper is to verify the tendency of theoretical results, but not to achieve the theoretical limit. Therefore, we choose relatively simple generator polynomial of CC to accelerate simulations.
} 


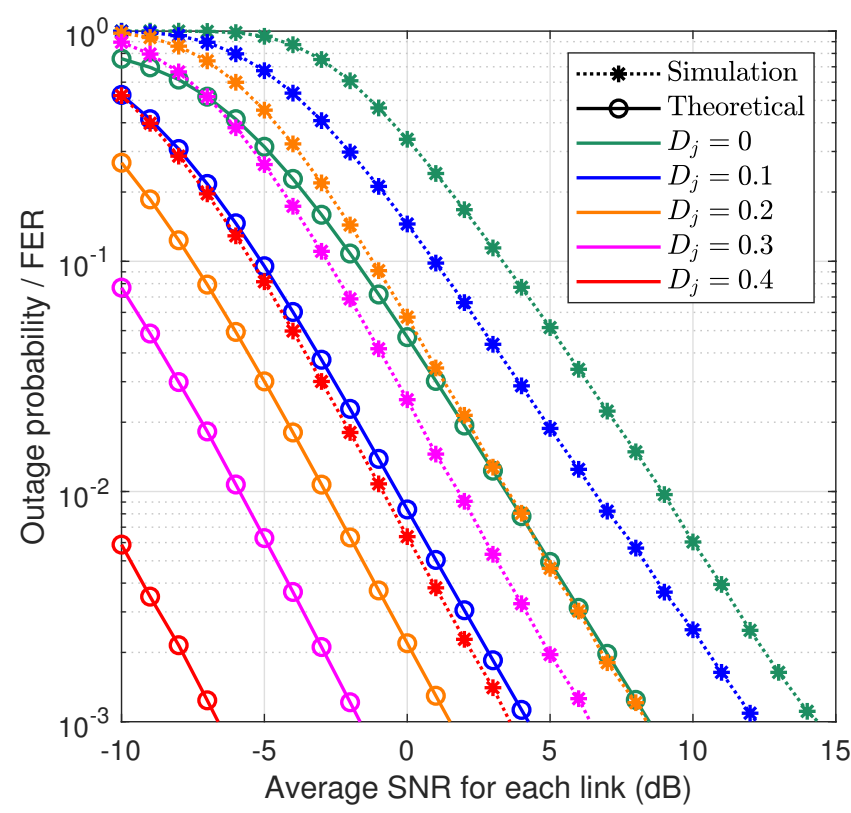

Fig. 13. Simulation results with $\rho=0.1$.

probability for less strict distortion requirements. The reason for the obvious gap includes two aspects, i.e., the relatively simple coding scheme, and incomplete utilization of joint typicality [28] in sequence-wise. First, it is even not easy to achieve Shannon limit for point-to-point communication. In addition, the theoretical inner bound is achieved by joint typicality coding scheme, which jointly reconstructs estimates in sequence-wise. However, the joint decoder in simulation exchanges the extrinsic information in bit-wise, which cannot completely exploit the joint typicality among the source sequences and the helper sequence. This observation inspires the necessity to develop a joint decoding algorithm for exchanging extrinsic information in sequence-wise.

\section{CONCLUSION}

In this work, we have analyzed the performance of lossy communications with a helper in terms of rate-distortion and outage probability over independent block Rayleigh fading channels. Based on Shannon's lossy source-channel separation theorem, we start the performance analysis from multiterminal source coding with a helper, and then take the channel conditions into consideration in the derivation of outage probability. For the rate-distortion analysis, we derive an inner bound on the achievable rate-distortion region for multiterminal source coding with two binary sources and one helper. The theoretical results demonstrate that a helper can obviously enlarge the achievable rate-distortion region; moreover, the inner bound derived in this paper coincides with the Berger-Tung inner bound when the helper rate decreases to 0 , i.e., the helper link is equivalently removed. Then, we apply the derived inner bound into the outage probability analysis. In order to briefly calculate the outage probability, the area outside the achievable rate-distortion region is divided into several subareas. Moreover, we investigate the performance improvement by introducing a helper with respect to the outage probability. The curves of outage probability indicate that a helper can reduce the outage probability, and make the system achieve higher order of diversity. Finally, we design a joint decoding algorithm for the helper structure being XOR, and conduct a series of simulations to evaluate the practical performance of lossy communications with a helper. The simulation results verify the second order diversity observed in the theoretical analysis, and the reduction of outage probability by increasing the acceptable distortions.

\section{REFERENCES}

[1] B. Ahlgren, M. Hidell, and E. C.-H. Ngai, "Internet of Things for smart cities: Interoperability and open data," IEEE Internet Computing, vol. 20, no. 6, pp. 52-56, Dec. 2016.

[2] A. Irawan and T. Matsumoto, "Feedback-assisted correlated packet transmission with a helper," IEEE Transactions on Vehicular Technology, May 2018.

[3] R. Tandon, S. Ulukus, and K. Ramchandran, "Secure source coding with a helper," IEEE Transactions on Information Theory, vol. 59, no. 4, pp. 2178-2187, Oct. 2013.

[4] P. Ostovari, J. Wu, and A. Khreishah, "Cooperative internet access using helper nodes and opportunistic scheduling," IEEE Transactions on Vehicular Technology, vol. 66, no. 7, pp. 6439-6448, Jul. 2017.

[5] S. Gherekhloo and A. Sezgin, "Latency-limited broadcast channel with cache-equipped helpers," IEEE Transactions on Wireless Communications, Jul. 2017.

[6] R. Joda and F. Lahouti, "Network code design for orthogonal two-hop network with broadcasting relay: A joint source-channel-network coding approach," IEEE Transactions on Communications, vol. 60, no. 1, pp. 132-142, Jan. 2012.

[7] J. He, V. Tervo, S. Qian, Q. Xue, M. Juntti, and T. Matsumoto, "Performance analysis of lossy decode-and-forward for non-orthogonal MARCs," IEEE Transactions on Wireless Communications, vol. 17, no. 3, pp. 1545-1558, Mar. 2018.

[8] X. Zhou, M. Cheng, X. He, and T. Matsumoto, "Exact and approximated outage probability analyses for decode-and-forward relaying system allowing intra-link errors," IEEE Transactions on Wireless Communications, vol. 13, no. 12, pp. 7062-7071, Dec. 2014.

[9] J. He, V. Tervo, X. Zhou, X. He, S. Qian, M. Cheng, M. Juntti, and T. Matsumoto, "A tutorial on lossy forwarding cooperative relaying," IEEE Communications Surveys \& Tutorials, vol. 21, no. 1, pp. 66-87, Firstquarter 2018.

[10] C. E. Shannon, "A mathematical theory of communication," Bell System Technical Journal, vol. 27, no. 3, pp. 379-423, Jul. 1948.

[11] _ _ "Coding theorems for a discrete source with a fidelity criterion," IRE Nat. Conv. Rec, vol. 4, no. 142-163, p. 1, Mar. 1959.

[12] J.-J. Xiao and Z.-Q. Luo, "Multiterminal source-channel communication over an orthogonal multiple-access channel," IEEE Transactions on Information Theory, vol. 53, no. 9, pp. 3255-3264, Sep. 2007.

[13] D. Slepian and J. Wolf, "Noiseless coding of correlated information sources," IEEE Transactions on Information Theory, vol. 19, no. 4, pp. 471-480, Jul. 1973.

[14] T. Berger, "Multiterminal source coding," in The Information Theory Approach to Communications, G. Longo, Ed. New York: SpringerVerlag, 1978, pp. 171-231.

[15] S. Y. Tung, "Multiterminal source coding," Ph.D. dissertation, School of Electrical Engineering, Cornell University, Ithaca, New York, 1978.

[16] X. He, X. Zhou, P. Komulainen, M. Juntti, and T. Matsumoto, "A lower bound analysis of hamming distortion for a binary CEO problem with joint source-channel coding," IEEE Transactions on Communications, vol. 64, no. 1, pp. 343-353, Jan. 2016.

[17] X. He, X. Zhou, M. Juntti, and T. Matsumoto, "A rate-distortion region analysis for a binary CEO problem," in IEEE 83rd Vehicular Technology Conference (VTC Spring), Nanjing, China, May 2016, pp. 1-5.

[18] R. Ahlswede and J. Korner, "Source coding with side information and a converse for degraded broadcast channels," IEEE Transactions on Information Theory, vol. 21, no. 6, pp. 629-637, Nov. 1975.

[19] A. Wyner and J. Ziv, "The rate-distortion function for source coding with side information at the decoder," IEEE Transactions on information Theory, vol. 22, no. 1, pp. 1-10, Jan. 1976. 
[20] A. Sechelea, A. Munteanu, S. Cheng, and N. Deligiannis, "On the ratedistortion function for binary source coding with side information," IEEE Transactions on Communications, vol. 64, no. 12, pp. 5203-5216, Dec. 2016.

[21] A. B. Wagner and V. Anantharam, "An improved outer bound for multiterminal source coding," IEEE Transactions on Information Theory, vol. 54, no. 5, pp. 1919-1937, May 2008.

[22] S. Jana and R. Blahut, "Canonical description for multiterminal source coding," in IEEE International Symposium on Information Theory (ISIT), Toronto, ON, Canada, Jul. 2008, pp. 697-701.

[23] R. Timo, T. Chan, and A. Grant, "Rate distortion with side-information at many decoders," IEEE Transactions on Information Theory, vol. 57, no. 8, pp. 5240-5257, Aug. 2011.

[24] J. N. Laneman, D. N. Tse, and G. W. Wornell, "Cooperative diversity in wireless networks: Efficient protocols and outage behavior," IEEE Transactions on Information Theory, vol. 50, no. 12, pp. 3062-3080, 2004.

[25] X. Zhou, P.-S. Lu, K. Anwar, and T. Matsumoto, "Correlated sources transmission in orthogonal multiple access relay channel: Theoretical analysis and performance evaluation," IEEE Transactions on Wireless Communications, vol. 13, no. 3, pp. 1424-1435, Jan. 2014.

[26] P.-S. Lu, X. Zhou, and T. Matsumoto, "Outage probabilities of orthogonal multiple-access relaying techniques with imperfect source-relay links," IEEE Transactions on Wireless Communications, vol. 14, no. 4, pp. 2269-2280, Dec. 2015.

[27] W. Lin and T. Matsumoto, "An analysis of performance improvement by a helper for wireless sensor networks," in IEEE 29th Annual International Symposium on Personal, Indoor and Mobile Radio Communications (PIMRC), Bologna, Italy, Sep. 2018.

[28] A. El Gamal and Y.-H. Kim, Network information theory. Cambridge University Press, 2011.

[29] K. Anwar and T. Matsumoto, "Accumulator-assisted distributed turbo codes for relay systems exploiting source-relay correlation," IEEE Communications Letters, vol. 16, no. 7, pp. 1114-1117, Jul. 2012.

[30] J. Garcia-Frias and Y. Zhao, "Near-Shannon/Slepian-Wolf performance for unknown correlated sources over AWGN channels," IEEE Transactions on Communications, vol. 53, no. 4, pp. 555-559, Apr. 2005.

[31] X. Zhou, X. He, K. Anwar, and T. Matsumoto, "GREAT-CEO: larGe scale distRibuted dEcision mAking Technique for wireless Chief Executive Officer problems," IEICE Transactions on Communications, vol. 95, no. 12, pp. 3654-3662, Dec. 2012.

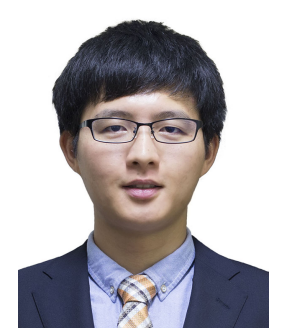

Wensheng Lin (S'17) received the B.Eng. degree in communication engineering, and the M.Eng. degree in electronic and communication engineering from Northwestern Polytechnical University, Xi'an, China, in 2013 and 2016, respectively. He is currently pursuing the Ph.D. degree at the School of Information Science, Japan Advanced Institute of Science and Technology (JAIST), Ishikawa, Japan. His research interests include network information theory, distributed source coding, helper structure design, and optimal encoding/decoding design.

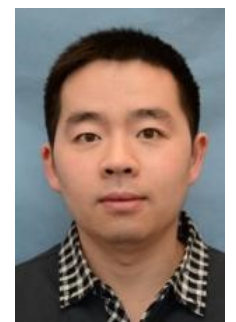

Qiang Xue (S'09-M'17) received the M.Sc. degree in communications engineering from University of York, UK, in 2008, and the Dr.Sc. degree in communications engineering from University of Oulu, Finland, in 2016. He is currently working as a post-doc researcher at the Centre for Wireless Communications, University of Oulu. His research interests span information theory, signal processing, and cooperative communications.
Jiguang He (S'16) received the B.Eng. degree from Harbin Institute of Technology, Harbin, China, in 2010, M.Sc. degree from Xiamen University, Xiamen, China, in 2013, and Dr. Sc degree from Unviersity of Oulu, Oulu, Finland, in 2018, all in communications engineering. From September 2013 to March 2015, he was with Key Laboratory of Millimeter Waves at City University of Hong Kong, conducting research on channel tracking over millimeter wave MIMO systems. Since June 2015, he has been with Centre for Wireless Communications (CWC), University of Oulu, Oulu, Finland. His research interests span cooperative communications, network information theory, joint source and channel coding, and distributed compressive sensing.

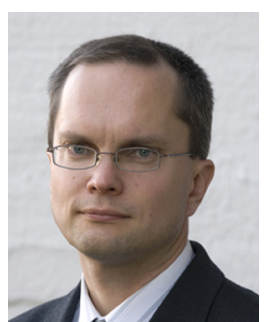

Markku Juntti (S'93-M'98-SM'04) received his M.Sc. (EE) and Dr.Sc. (EE) degrees from University of Oulu, Oulu, Finland in 1993 and 1997, respectively.

Dr. Juntti was with University of Oulu in 1992-98. In academic year 1994-95, he was a Visiting Scholar at Rice University, Houston, Texas. In 1999-2000, he was a Senior Specialist with Nokia Networks. Dr. Juntti has been a professor of communications engineering since 2000 at University of Oulu, Centre for Wireless Communications (CWC), where he leads the Communications Signal Processing (CSP) Research Group. He also serves as Head of CWC - Radio Technologies (RT) Research Unit. His research interests include signal processing for wireless networks as well as communication and information theory. $\mathrm{He}$ is an author or co-author in some 350 papers published in international journals and conference records as well as in books WCDMA for UMTS and Signal Processing Handbook. Dr. Juntti is also an Adjunct Professor at Department of Electrical and Computer Engineering, Rice University, Houston, Texas, USA.

Dr. Juntti is an Editor of IEEE TRANSACTIONS ON COMMUNICATIONS and was an Associate Editor for IEEE TRANSACTIONS ON VEHICUlaR TECHNOLOGY in 2002-2008. He was Secretary of IEEE Communication Society Finland Chapter in 1996-97 and the Chairman for years 2000-01. He has been Secretary of the Technical Program Committee (TPC) of the 2001 IEEE International Conference on Communications (ICC 2001), and the Co-Chair of the Technical Program Committee of 2004 Nordic Radio Symposium and 2006 IEEE International Symposium on Personal, Indoor and Mobile Radio Communications (PIMRC 2006), and the General Chair of 2011 IEEE Communication Theory Workshop (CTW 2011). He has served as CoChair of the Signal Processing for Communications Symposium of Globecom 2014 Signal Processing for Communications Symposium and IEEE GlobalSIP 2016 Symposium on Transceivers and Signal Processing for 5G Wireless and mm-Wave Systems, and ACM NanoCom 2018. 


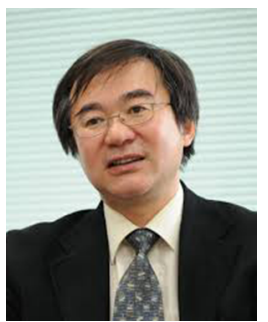

Tad Matsumoto (S'84-SM'95-F'10) received his B.S., M.S., degrees, both under the mentorship by Prof. Shin-Ichi Takahashi, and Ph.D. degree under the supervision by Prof. Masao Nakagawa, from Keio University, Yokohama, Japan, in 1978, 1980, and 1991, respectively, all in electrical engineering.

He joined Nippon Telegraph and Telephone Corporation (NTT) in April 1980. Since he engaged in NTT, he was involved in a lot of research and development projects, all for mobile wireless communications systems. In July 1992, he transferred to NTT DoCoMo, where he researched Code-Division Multiple-Access techniques for Mobile Communication Systems. In April 1994, he transferred to NTT America, where he served as a Senior Technical Advisor of a joint project between NTT and NEXTEL Communications. In March 1996, he returned to NTT DoCoMo, where he served as a Head of the Radio Signal Processing Laboratory until August of 2001; He worked on adaptive signal processing, multiple-input multiple-output turbo signal detection, interference cancellation, and spacetime coding techniques for broadband mobile communications. In March 2002, he moved to University of Oulu, Finland, where he served as a Professor at Centre for Wireless Communications. In 2006, he served as a Visiting Professor at Ilmenau University of Technology, Ilmenau, Germany, funded by the German MERCATOR Visiting Professorship Program. Since April 2007, he has been serving as a Professor at Japan Advanced Institute of Science and Technology (JAIST), Japan, while also keeping a cross-appointment position at University of Oulu.

He has led a lot of projects funded by Academy-of-Finland, European FP7, and Japan Society for the Promotion of Science as well as by Japanese private companies.

Prof. Matsumoto has been appointed as a Finland Distinguished Professor for a period from January 2008 to December 2012, funded by the Finnish National Technology Agency (Tekes) and Finnish Academy, under which he preserves the rights to participate in and apply to European and Finnish national projects. Prof. Matsumoto is a recipient of IEEE VTS Outstanding Service Award (2001), Nokia Foundation Visiting Fellow Scholarship Award (2002), IEEE Japan Council Award for Distinguished Service to the Society (2006), IEEE Vehicular Technology Society James R. Evans Avant Garde Award (2006), and Thuringen State Research Award for Advanced Applied Science (2006), 2007 Best Paper Award of Institute of Electrical, Communication, and Information Engineers of Japan (2008), Telecom System Technology Award by the Telecommunications Advancement Foundation (2009), IEEE Communication Letters Exemplary Reviewer (2011), Nikkei Wireless Japan Award (2013), IEEE VTS Recognition for Outstanding Distinguished Lecturer (2016), and IEEE Transactions on Communications Exemplary Reviewer (2018). He is a Fellow of IEEE and a Member of IEICE. He is serving as an IEEE Vehicular Technology Distinguished Speaker since July 2016. 